\title{
Short- and long-range corrected hybrid density functionals with the D3 dispersion corrections
}

\author{
Chih-Wei Wang, ${ }^{1}$ Kerwin Hui, ${ }^{1}$ and Jeng-Da Chai ${ }^{1,2, *}$ \\ ${ }^{1}$ Department of Physics, National Taiwan University, Taipei 10617, Taiwan \\ ${ }^{2}$ Center for Theoretical Sciences and Center for Quantum Science and Engineering, \\ National Taiwan University, Taipei 1061\%, Taiwan
}

(Dated: July 1, 2018)

\begin{abstract}
We propose a short- and long-range corrected (SLC) hybrid scheme employing 100\% HartreeFock (HF) exchange at both zero and infinite interelectronic distances, wherein three SLC hybrid density functionals with the D3 dispersion corrections (SLC-LDA-D3, SLC-PBE-D3, and SLC-B97D3) are developed. SLC-PBE-D3 and SLC-B97-D3 are shown to be accurate for a very diverse range of applications, such as core ionization and excitation energies, thermochemistry, kinetics, noncovalent interactions, dissociation of symmetric radical cations, vertical ionization potentials, vertical electron affinities, fundamental gaps, and valence, Rydberg, and long-range charge-transfer excitation energies. Relative to $\omega$ B97X-D, SLC-B97-D3 provides significant improvement for core ionization and excitation energies and noticeable improvement for the self-interaction, asymptote, energy-gap, and charge-transfer problems, while performing similarly for thermochemistry, kinetics, and noncovalent interactions.
\end{abstract}

\footnotetext{
* Author to whom correspondence should be addressed. Electronic mail: jdchai@phys.ntu.edu.tw
} 


\section{INTRODUCTION}

Due to its decent balance between cost and performance, Kohn-Sham density functional theory (KS-DFT) [1, 2] has been a very popular electronic structure method for studying the ground-state properties of large systems [3-6]. Recently, one of its most important extensions, time-dependent density functional theory (TDDFT) [7], has also been actively developed for studying the excited-state and time-dependent properties of large systems $[8-$ 14]. Nonetheless, the exact exchange-correlation $(\mathrm{XC})$ energy functional $E_{x c}[\rho]$, which is the essential ingredient of both KS-DFT and adiabatic TDDFT, has not been found, and hence, density functional approximations (DFAs) for $E_{x c}[\rho]$ have been successively developed to improve the accuracy of KS-DFT and TDDFT for general applications.

Functionals based on the conventional DFAs, such as the local density approximation (LDA) [15, 16], generalized gradient approximations (GGAs) [17], and meta-GGAs (MGGAs) [18, 19], are semilocal density functionals [20]. They are reasonably accurate for the properties governed by short-range $\mathrm{XC}$ effects, and are computationally favorable for very large systems. Nevertheless, owing to the inadequate treatment of nonlocal XC effects [36, 21] , semilocal density functionals can perform very poorly for the problems related to the self-interaction error (SIE) [22], noncovalent interaction error (NCIE) [23-25], and static correlation error (SCE) [26 32$]$.

In particular, some of these situations happen in the asymptotic regions $(r \rightarrow \infty)$ of molecules, where the electron densities decay exponentially. In these regions, owing to the pronounced SIEs associated with semilocal density functionals, the functional derivatives of most semilocal density functionals (i.e., the semilocal XC potentials) do not exhibit the correct $(-1 / r)$ decay. Consequently, most semilocal density functionals can yield erroneous results for the highest occupied molecular orbital (HOMO) energies [33 38] and high-lying Rydberg excitation energies [11, 39-41]. Even if the asymptote problems can be properly resolved by the recently developed semilocal density functionals with correct asymptotic behavior [42 46] and asymptotically corrected model XC potentials [47 51], the SIE prob-

lems may remain unresolved [52]. Besides, semilocal density functionals are inaccurate for charge-transfer (CT) excitation energies [41, 42, 52 61], due to the lack of a space- and frequency-dependent discontinuity in the adiabatic XC kernel adopted in TDDFT [62].

In 1993, on the basis of the adiabatic-connection formalism, Becke proposed global hybrid 
density functionals [63, 64], combining semilocal density functionals with a small fraction (typically ranging from 0.2 to 0.25 for thermochemistry, and from 0.4 to 0.6 for kinetics) of Hartree-Fock (HF) exchange [63 72]. However, in certain situations, especially in the asymptotic regions of molecular systems, a very large fraction (even 100\%) of HF exchange is needed. Widely used global hybrid density functionals, such as B3LYP [64, 65], PBE0 [68, 69], and M06-2X [71], do not qualitatively resolve the SIE, asymptote, and CT problems [52, 73$]$.

With the aim of resolving these problems, long-range corrected (LC) hybrid density functionals [74 87] have recently received considerable attention. A commonly used LC hybrid density functional (e.g., LC- $\omega$ PBE [77] and $\omega$ B97 [79]) employs 100\% HF exchange for the long-range (LR) part of the interelectronic repulsion operator $\operatorname{erf}\left(\omega r_{12}\right) / r_{12}$, a semilocal exchange for the complementary short-range (SR) operator $\operatorname{erfc}\left(\omega r_{12}\right) / r_{12}$, and a semilocal correlation for the entire Coulomb operator $1 / r_{12}$, with the parameter $\omega$ (typically ranging from 0.2 to $0.5 \mathrm{bohr}^{-1}$ ) specifying the partitioning of the interelectronic distance $r_{12}=$ $\left|\mathbf{r}_{1}-\mathbf{r}_{2}\right|$ (atomic units are used throughout this paper). Here, erf is the standard error function, and erfc is the complementary error function. Besides, the inclusion of a small fraction of $\mathrm{HF}$ exchange at short range has been shown to improve the overall accuracy of conventional LC hybrid scheme (e.g., wB97X [79]). Over the years, LC hybrid functionals have been shown to qualitatively resolve the SIE, asymptote, and CT problems, offering a cost-effective way to incorporate nonlocal exchange effects.

To properly account for noncovalent interactions, an accurate description of middle- and long-range dynamical correlation effects is essential. Accordingly, LC hybrid functionals can be combined with the DFT-D (KS-DFT with empirical dispersion corrections) schemes [24, 88 92] (e.g., $\omega$ B97X-D [80], $\omega \mathrm{M} 05-\mathrm{D}$ [84], $\omega \mathrm{M} 06-\mathrm{D} 3$ [85], and $\omega$ B97X-D3 [85]) and the double-hybrid (adding a small fraction of second-order Møller-Plesset correlation) schemes [72, 93 105] (e.g., wB97X-2 [82]). Alternatively, LC hybrid functionals can also be incorporated with a fully nonlocal correlation density functional for van der Waals interactions (vdW-DF) [106 108] (e.g., $\omega$ B97X-V [86] and $\omega$ B97M-V [87]). Recently, we have shown that the $\omega \mathrm{B} 97$ series of functionals ( $\omega$ B97, $\omega$ B97X, $\omega$ B97X-D, etc.) has yielded impressive accuracy for various applications [52, 73, 109], such as thermochemistry, kinetics, noncovalent interactions, dissociation of symmetric radical cations, frontier orbital energies, fundamental gaps, and valence, Rydberg, and long-range CT excitation energies. 
In spite of its general applicability, there are some situations, however, where the $\omega$ B97 series can fail qualitatively. Very recently, Maier et al. [110] have shown that popular LC hybrid functionals, such as LC- $\omega$ PBE and $\omega$ B97X-D, perform very poorly for core excitation energies. They have also shown that global hybrid functionals with a large fraction (about $50 \%$ ) of HF exchange perform reasonably well for core excitation energies, showing consistency with the previous findings of Nakai and co-workers [111, 112]. However, global hybrid functionals with $50 \% \mathrm{HF}$ exchange may not consistently perform well for thermochemistry and many other properties that do not require a large fraction of HF exchange. Within the framework of LC hybrid scheme, Hirao and co-workers have shown that the fraction of HF exchange at short range should be responsible for an accurate description of core excitation energies [113]. Similarly, the short-range corrected hybrid density functionals proposed by Besley et al. have been shown to accurately describe core excitation energies [114].

On the other hand, Chai and Head-Gordon have shown that the fraction of HF exchange in the middle-range (MR) region $\left(0.5\right.$ bohr $\lesssim r_{12} \lesssim 1.5$ bohr) is important for a good balanced description of thermochemistry and kinetics [115]. Besides, they have argued that the fraction of HF exchange in the LR region $\left(r_{12} \gtrsim 1.5\right.$ bohr $)$ should be crucial for the properties sensitive to the tail contributions (e.g., the SIE, asymptote, and CT problems), and the fraction of HF exchange in the SR region $\left(r_{12} \lesssim 0.5\right.$ bohr $)$ should be responsible for the properties involving changes in the core contributions to $E_{x c}[\rho]$, such as core excitation energies. However, the SR region of the HF exchange operators adopted in the $\omega$ B97 series has not been fully explored. Note that the fraction of HF exchange at zero interelectronic distance $r_{12}=0$ is only $0.00,0.16,0.22$, and 0.20 for $\omega \mathrm{B} 97, \omega \mathrm{B} 97 \mathrm{X}, \omega \mathrm{B} 97 \mathrm{X}-\mathrm{D}$, and $\omega \mathrm{B} 97 \mathrm{X}$ D3, respectively. Nonetheless, as the electron densities in the core region are rather high (i.e., close to the high-density limit, where HF exchange should dominate correlation), we argue that a very large fraction of HF exchange in the SR region should be adopted for an accurate description of the properties sensitive to the core contributions (e.g., core ionization and excitation energies).

In this work, we intend to improve the performance of the widely used LC hybrid functionals, LC- $\omega \mathrm{PBE}$ and the $\omega \mathrm{B} 97$ series, for core ionization and excitation energies, while retaining similar accuracy for many other applications. Specifically, we propose a new LC hybrid scheme employing $100 \% \mathrm{HF}$ exchange at $r_{12}=0$ (i.e., the LC hybrid scheme is also short-range corrected), which is in strong contrast to the popular LC hybrid scheme (i.e., 
with the erf operator) and other LC hybrid schemes (e.g., with the erfgau [113, 116 118] and terf [119, 120] operators) employing vanishing HF exchange at $r_{12}=0$. The rest of this paper is organized as follows. We describe the short- and long-range corrected (SLC) hybrid scheme in Section II, and develop three SLC hybrid density functionals with the D3 dispersion corrections in Section III. The performance of our new functionals is compared with other functionals in Section IV (on the training set), and in Section V (on various test sets). Our conclusions are given in Section VI.

\section{SHORT- AND LONG-RANGE CORRECTED (SLC) HYBRID SCHEME}

In the SLC hybrid scheme, we first define the SLR operator $f_{\mathrm{SLR}}\left(r_{12}\right) / r_{12}$, which is an operator that approaches $1 / r_{12}$ at both the SR $\left(r_{12}=0\right)$ and $\operatorname{LR}\left(r_{12} \rightarrow \infty\right)$ limits, and the complementary MR operator $f_{\mathrm{MR}}\left(r_{12}\right) / r_{12}=\left(1-f_{\mathrm{SLR}}\left(r_{12}\right)\right) / r_{12}$ to partition the Coulomb operator:

$$
\frac{1}{r_{12}}=\frac{f_{\mathrm{SLR}}\left(r_{12}\right)}{r_{12}}+\frac{f_{\mathrm{MR}}\left(r_{12}\right)}{r_{12}}
$$

In this work, we adopt

$$
f_{\mathrm{SLR}}\left(r_{12}\right)=\operatorname{erfc}\left(\omega_{\mathrm{SR}} r_{12}\right)+\operatorname{erf}\left(\omega_{\mathrm{LR}} r_{12}\right)
$$

as a simple sum of the SR function $\operatorname{erfc}\left(\omega_{\mathrm{SR}} r_{12}\right)$ and $\mathrm{LR}$ function $\operatorname{erf}\left(\omega_{\mathrm{LR}} r_{12}\right)$. Here, $\omega_{\mathrm{SR}}$ and $\omega_{\mathrm{LR}}$ are parameters controlling the SR and LR behavior, respectively, of $f_{\mathrm{SLR}}\left(r_{12}\right)$. Accordingly, we have

$$
\begin{aligned}
f_{\mathrm{MR}}\left(r_{12}\right) & =1-\left(\operatorname{erfc}\left(\omega_{\mathrm{SR}} r_{12}\right)+\operatorname{erf}\left(\omega_{\mathrm{LR}} r_{12}\right)\right) \\
& =\operatorname{erfc}\left(\omega_{\mathrm{LR}} r_{12}\right)-\operatorname{erfc}\left(\omega_{\mathrm{SR}} r_{12}\right) .
\end{aligned}
$$

After the SLR/MR partition, a SLC hybrid density functional is defined as

$$
E_{x c}^{\mathrm{SLC}}=E_{x}^{\mathrm{SLR}-\mathrm{HF}}+E_{x}^{\mathrm{MR}-\mathrm{DFA}}+E_{c}^{\mathrm{DFA}} .
$$

Here, $E_{c}^{\mathrm{DFA}}$ is the DFA correlation energy of the Coulomb operator $1 / r_{12}, E_{x}^{\mathrm{SLR}-\mathrm{HF}}$ is the HF exchange energy of the SLR operator $f_{\mathrm{SLR}}\left(r_{12}\right) / r_{12}=\operatorname{erfc}\left(\omega_{\mathrm{SR}} r_{12}\right) / r_{12}+\operatorname{erf}\left(\omega_{\mathrm{LR}} r_{12}\right) / r_{12}$ (computed by the occupied Kohn-Sham (KS) orbitals $\left.\left\{\psi_{i \sigma}(\mathbf{r})\right\}\right)$ :

$$
\begin{aligned}
E_{x}^{\mathrm{SLR}-\mathrm{HF}} & =-\frac{1}{2} \sum_{\sigma}^{\alpha, \beta} \sum_{i, j}^{o c c .} \iint \psi_{i \sigma}^{*}\left(\mathbf{r}_{1}\right) \psi_{j \sigma}^{*}\left(\mathbf{r}_{2}\right) \frac{f_{\mathrm{SLR}}\left(r_{12}\right)}{r_{12}} \psi_{j \sigma}\left(\mathbf{r}_{1}\right) \psi_{i \sigma}\left(\mathbf{r}_{2}\right) d \mathbf{r}_{1} d \mathbf{r}_{2} \\
& =E_{x}^{\mathrm{SR}-\mathrm{HF}}\left(\omega_{\mathrm{SR}}\right)+E_{x}^{\mathrm{LR}-\mathrm{HF}}\left(\omega_{\mathrm{LR}}\right),
\end{aligned}
$$


where

$$
E_{x}^{\mathrm{SR}-\mathrm{HF}}\left(\omega_{\mathrm{SR}}\right)=-\frac{1}{2} \sum_{\sigma}^{\alpha, \beta} \sum_{i, j}^{o c c .} \iint \psi_{i \sigma}^{*}\left(\mathbf{r}_{1}\right) \psi_{j \sigma}^{*}\left(\mathbf{r}_{2}\right) \frac{\operatorname{erfc}\left(\omega_{\mathrm{SR}} r_{12}\right)}{r_{12}} \psi_{j \sigma}\left(\mathbf{r}_{1}\right) \psi_{i \sigma}\left(\mathbf{r}_{2}\right) d \mathbf{r}_{1} d \mathbf{r}_{2}
$$

is the HF exchange energy of the SR operator $\operatorname{erfc}\left(\omega_{\mathrm{SR}} r_{12}\right) / r_{12}$, and

$$
E_{x}^{\mathrm{LR}-\mathrm{HF}}\left(\omega_{\mathrm{LR}}\right)=-\frac{1}{2} \sum_{\sigma}^{\alpha, \beta} \sum_{i, j}^{\text {occ. }} \iint \psi_{i \sigma}^{*}\left(\mathbf{r}_{1}\right) \psi_{j \sigma}^{*}\left(\mathbf{r}_{2}\right) \frac{\operatorname{erf}\left(\omega_{\mathrm{LR}} r_{12}\right)}{r_{12}} \psi_{j \sigma}\left(\mathbf{r}_{1}\right) \psi_{i \sigma}\left(\mathbf{r}_{2}\right) d \mathbf{r}_{1} d \mathbf{r}_{2}
$$

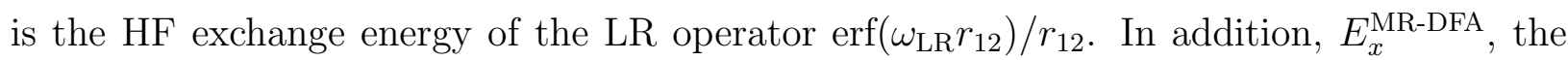
DFA exchange energy of the MR operator $f_{\mathrm{MR}}\left(r_{12}\right) / r_{12}=\operatorname{erfc}\left(\omega_{\mathrm{LR}} r_{12}\right) / r_{12}-\operatorname{erfc}\left(\omega_{\mathrm{SR}} r_{12}\right) / r_{12}$, can be expressed as

$$
E_{x}^{\mathrm{MR}-\mathrm{DFA}}=E_{x}^{\mathrm{SR}-\mathrm{DFA}}\left(\omega_{\mathrm{LR}}\right)-E_{x}^{\mathrm{SR}-\mathrm{DFA}}\left(\omega_{\mathrm{SR}}\right)
$$

where $E_{x}^{\mathrm{SR}-\mathrm{DFA}}(\omega)$ is the DFA exchange energy of the SR operator $\operatorname{erfc}\left(\omega r_{12}\right) / r_{12}$.

On the basis of Eq. (5), $f_{\mathrm{SLR}}\left(r_{12}\right)$ can be regarded as the fraction of HF exchange at $r_{12}$ for the SLC hybrid density functional. Therefore, we impose the constraint $0 \leq \omega_{\mathrm{LR}} \leq \omega_{\mathrm{SR}}<\infty$ to ensure that $0 \leq f_{\mathrm{MR}}\left(r_{12}\right) \leq 1$ and hence, $0 \leq f_{\mathrm{SLR}}\left(r_{12}\right) \leq 1$ can be satisfied at each $r_{12}$. For $\omega_{\mathrm{SR}}=\omega_{\mathrm{LR}}$, we have $f_{\mathrm{MR}}\left(r_{12}\right)=0$ and $f_{\mathrm{SLR}}\left(r_{12}\right)=1$, employing the full HF exchange and a DFA correlation. Note that $f_{\mathrm{SLR}}\left(r_{12}\right)$ (given by Eq. (2)) provides a smooth transition between the following two limits:

$$
f_{\mathrm{SLR}}\left(r_{12}=0\right)=1, \lim _{r_{12} \rightarrow \infty} f_{\mathrm{SLR}}\left(r_{12}\right)=1
$$

employing $100 \% \mathrm{HF}$ exchange at both the SR $\left(r_{12}=0\right)$ and $\operatorname{LR}\left(r_{12} \rightarrow \infty\right)$ limits. Note also that the SLC hybrid scheme reduces to the popular LC hybrid scheme (i.e., with the erf operator) as $\omega_{\mathrm{SR}} \rightarrow \infty$, while it reduces to pure KS-DFT as $\omega_{\mathrm{SR}} \rightarrow \infty$ and $\omega_{\mathrm{LR}}=0$.

\section{SLC HYBRID FUNCTIONALS WITH DISPERSION CORRECTIONS}

On the basis of Eq. (4), here we introduce three SLC hybrid density functionals with the D3 dispersion corrections. As the simplest DFA is the LDA, we define the SLC-LDA functional as

$$
E_{x c}^{\mathrm{SLC}-\mathrm{LDA}}=E_{x}^{\mathrm{SLR}-\mathrm{HF}}+E_{x}^{\mathrm{MR}-\mathrm{LDA}}+E_{c}^{\mathrm{LDA}}
$$


where $E_{c}^{\mathrm{LDA}}$ is the LDA correlation functional [16], $E_{x}^{\mathrm{SLR}-\mathrm{HF}}$ is the SLR-HF exchange energy (given by Eq. (5) ), and

$$
E_{x}^{\mathrm{MR}-\mathrm{LDA}}=E_{x}^{\mathrm{SR}-\mathrm{LDA}}\left(\omega_{\mathrm{LR}}\right)-E_{x}^{\mathrm{SR}-\mathrm{LDA}}\left(\omega_{\mathrm{SR}}\right)=\sum_{\sigma}^{\alpha, \beta} \int e_{x \sigma}^{\mathrm{MR}-\mathrm{LDA}} d \mathbf{r}
$$

is the MR-LDA exchange functional, which is known due to the analytical form of $E_{x}^{\mathrm{SR}-\mathrm{LDA}}(\omega)$, the LDA exchange functional of the SR operator $\operatorname{erfc}\left(\omega r_{12}\right) / r_{12}[74,121]$. Here, $e_{x \sigma}^{\mathrm{MR}-\mathrm{LDA}}$ is the MR-LDA exchange energy density for $\sigma$-spin,

$$
e_{x \sigma}^{\mathrm{MR}-\mathrm{LDA}}=-\frac{3}{2}\left(\frac{3}{4 \pi}\right)^{1 / 3} \rho_{\sigma}^{4 / 3}(\mathbf{r})\left[F\left(a_{\mathrm{LR}, \sigma}\right)-F\left(a_{\mathrm{SR}, \sigma}\right)\right],
$$

where $a_{\mathrm{LR}, \sigma} \equiv \omega_{\mathrm{LR}} /\left(2\left(6 \pi^{2} \rho_{\sigma}(\mathbf{r})\right)^{1 / 3}\right)$ and $a_{\mathrm{SR}, \sigma} \equiv \omega_{\mathrm{SR}} /\left(2\left(6 \pi^{2} \rho_{\sigma}(\mathbf{r})\right)^{1 / 3}\right)$ are dimensionless parameters controlling the values of the attenuation function $F(a)$,

$$
F(a)=1-\frac{8}{3} a\left[\sqrt{\pi} \operatorname{erf}\left(\frac{1}{2 a}\right)-3 a+4 a^{3}+\left(2 a-4 a^{3}\right) \exp \left(-\frac{1}{4 a^{2}}\right)\right] .
$$

To go beyond the simplest SLC-LDA, we define the SLC-PBE functional as

$$
E_{x c}^{\mathrm{SLC}-\mathrm{PBE}}=E_{x}^{\mathrm{SLR}-\mathrm{HF}}+E_{x}^{\mathrm{MR}-\mathrm{PBE}}+E_{c}^{\mathrm{PBE}}
$$

where $E_{c}^{\mathrm{PBE}}$ is the PBE correlation functional [17], $E_{x}^{\mathrm{SLR}-\mathrm{HF}}$ is the SLR-HF exchange energy (given by Eq. (51)), and

$$
E_{x}^{\mathrm{MR}-\mathrm{PBE}}=E_{x}^{\mathrm{SR}-\mathrm{PBE}}\left(\omega_{\mathrm{LR}}\right)-E_{x}^{\mathrm{SR}-\mathrm{PBE}}\left(\omega_{\mathrm{SR}}\right)
$$

is the MR-PBE exchange functional, with $E_{x}^{\mathrm{SR}-\mathrm{PBE}}(\omega)$ being the PBE exchange functional of the SR operator $\operatorname{erfc}\left(\omega r_{12}\right) / r_{12}$ [122].

To further improve upon SLC-PBE, we adopt flexible functional forms in Eq. (41). Similar to the B97 ansatz [67], we define the SLC-B97 functional as

$$
E_{x c}^{\mathrm{SLC}-\mathrm{B} 97}=E_{x}^{\mathrm{SLR}-\mathrm{HF}}+E_{x}^{\mathrm{MR}-\mathrm{B} 97}+E_{c}^{\mathrm{B} 97} .
$$

Here, $E_{c}^{\mathrm{B} 97}$ has the same functional form as the B97 correlation functional [67], which can be decomposed into same-spin $E_{c \sigma \sigma}^{B 97}$ and opposite-spin $E_{c \alpha \beta}^{B 97}$ components,

$$
E_{c}^{\mathrm{B} 97}=\sum_{\sigma}^{\alpha, \beta} E_{c \sigma \sigma}^{\mathrm{B} 97}+E_{c \alpha \beta}^{\mathrm{B} 97}
$$


Here,

$$
\begin{aligned}
E_{c \sigma \sigma}^{\mathrm{B} 97} & =\int e_{c \sigma \sigma}^{\mathrm{LDA}} \sum_{i=0}^{m} c_{c \sigma \sigma, i}\left(\frac{\gamma_{c \sigma \sigma} s_{\sigma}^{2}}{1+\gamma_{c \sigma \sigma} s_{\sigma}^{2}}\right)^{i} d \mathbf{r} \\
E_{c \alpha \beta}^{\mathrm{B} 97} & =\int e_{c \alpha \beta}^{\mathrm{LDA}} \sum_{i=0}^{m} c_{c \alpha \beta, i}\left(\frac{\gamma_{c \alpha \beta} s_{a v}^{2}}{1+\gamma_{c \alpha \beta} s_{a v}^{2}}\right)^{i} d \mathbf{r}
\end{aligned}
$$

where $\gamma_{c \sigma \sigma}=0.2, \gamma_{c \alpha \beta}=0.006, s_{a v}^{2}=\frac{1}{2}\left(s_{\alpha}^{2}+s_{\beta}^{2}\right)$, and $s_{\sigma}=\left|\nabla \rho_{\sigma}(\mathbf{r})\right| / \rho_{\sigma}^{4 / 3}(\mathbf{r})$. The correlation energy densities $e_{c \sigma \sigma}^{\mathrm{LDA}}=e_{c}^{\mathrm{LDA}}\left(\rho_{\sigma}, 0\right)$ and $e_{c \alpha \beta}^{\mathrm{LDA}}=e_{c}^{\mathrm{LDA}}\left(\rho_{\alpha}, \rho_{\beta}\right)-e_{c}^{\mathrm{LDA}}\left(\rho_{\alpha}, 0\right)-e_{c}^{\mathrm{LDA}}\left(0, \rho_{\beta}\right)$ are derived from the PW92 parametrization of the LDA correlation energy density $e_{c}^{\mathrm{LDA}}\left(\rho_{\alpha}, \rho_{\beta}\right)$ [16], using the approach of Stoll et al. [123]. In addition, $E_{x}^{\mathrm{SLR}-\mathrm{HF}}$ is the SLR-HF exchange energy (given by Eq. (50)), and

$$
E_{x}^{\mathrm{MR}-\mathrm{B} 97}=\sum_{\sigma}^{\alpha, \beta} \int e_{x \sigma}^{\mathrm{MR}-\mathrm{LDA}} \sum_{i=0}^{m} c_{x \sigma, i}\left(\frac{\gamma_{x \sigma} s_{\sigma}^{2}}{1+\gamma_{x \sigma} s_{\sigma}^{2}}\right)^{i} d \mathbf{r}
$$

is the MR-B97 exchange functional, where $\gamma_{x \sigma}=0.004$ and $e_{x \sigma}^{\mathrm{MR}-\mathrm{LDA}}$ is given by Eq. (12). Note that $E_{x}^{\mathrm{MR}-\mathrm{B} 97}$ has the same functional form as the SR-B97 exchange functional (see Eq. (11) of Ref. [79]) when $\omega_{\mathrm{SR}} \rightarrow \infty$, and has the same functional form as the B97 exchange functional [67] when $\omega_{\mathrm{SR}} \rightarrow \infty$ and $\omega_{\mathrm{LR}}=0$.

Following the DFT-D3 scheme [90], our total energy is given by

$$
E_{\mathrm{DFT}-\mathrm{D} 3}=E_{\mathrm{KS}-\mathrm{DFT}}+E_{\mathrm{disp}}(\mathrm{D} 3)
$$

where $E_{\mathrm{KS}-\mathrm{DFT}}$ is the total energy in KS-DFT, and

$$
E_{\text {disp }}(\mathrm{D} 3)=-\sum_{n=6,8} \sum_{A>B} \frac{C_{n}^{A B}}{R_{A B}^{n}\left[1+6\left(s_{r, n} R_{0}^{A B} / R_{A B}\right)^{n+8}\right]}
$$

is the D3 dispersion correction (the unscaled version is adopted, and the three-body term is not included). Here, the second sum is over all atom pairs in the system, and $R_{A B}$ is the interatomic distance of atom pair $A B$, while the cutoff radius $R_{0}^{A B}$ and the dispersion coefficients $\left(C_{6}^{A B}\right.$ and $\left.C_{8}^{A B}\right)$ for atom pair $A B$ are provided in the DFT-D3 scheme [90]. Therefore, $s_{r, 6}$ and $s_{r, 8}$, which control the strength of dispersion correction, are the parameters to be determined.

In this work, the SLC-LDA (Eq. (10)), SLC-PBE (Eq. (14)), and SLC-B97 (Eq. (16)) functionals with the D3 dispersion corrections (Eq. (22) ) are denoted as SLC-LDA-D3, SLCPBE-D3, and SLC-B97-D3, respectively. Note that SLC-LDA-D3 and SLC-PBE-D3 satisfy 
the exact uniform electron gas (UEG) limit by construction, while the exact UEG limit for SLC-B97-D3 is enforced by imposing the following constraints: $c_{x \sigma, 0}=c_{c \sigma \sigma, 0}=c_{c \alpha \beta, 0}=1$.

The four parameters $\left(\omega_{\mathrm{SR}}, \omega_{\mathrm{LR}}, s_{r, 6}\right.$, and $\left.s_{r, 8}\right)$ of SLC-LDA-D3 and SLC-PBE-D3 are determined by least-squares fittings to the accurate experimental and theoretical data in the training set, involving

- the 223 atomization energies (AEs) of the G3/99 set [124],

- the 40 ionization potentials (IPs), 25 electron affinities (EAs), and 8 proton affinities (PAs) of the G2-1 set [125],

- the 76 barrier heights of the NHTBH38/04 and HTBH38/04 sets [126],

- the 22 noncovalent interactions of the S22 set [127, 128].

For the S22 set, an updated version of reference values from S22B [128] are adopted. For the parameter optimization, we focus on a range of possible $\omega_{\mathrm{SR}}(0.8,1.0,1.5,2.0,2.5$, 3.0, 3.5, and $\left.4.0 \mathrm{bohr}^{-1}\right)$ and $\omega_{\mathrm{LR}}\left(0.30,0.35,0.40,0.45\right.$, and $\left.0.50 \mathrm{bohr}^{-1}\right)$ values, and optimize the corresponding $s_{r, 6}$ and $s_{r, 8}$ in steps of 0.001 , for $0<s_{r, 6}<2$ and $0<s_{r, 8}<2$, respectively. The S22 data are weighted 10 times more than the others. As is usual in hybrid density functional approaches, the electronic energy is minimized with respect to the orbitals. Detailed information about the training set can be found in Refs. [79, 84, 85].

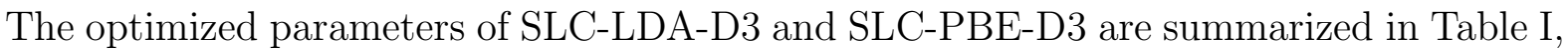
and the HF exchange operators adopted in SLC-LDA-D3, SLC-PBE-D3, and the $\omega$ B97 series are plotted in Figure 1. Note that the HF exchange operators adopted in LC- $\omega$ PBE and $\omega$ B97 are the same. As can be seen, the fractions of HF exchange adopted in SLCPBE-D3 and the $\omega$ B97 series are similar in the MR region, showing consistency with the previous findings of Chai and Head-Gordon [115] that the fine details of the MR region of the HF exchange operators adopted are important for good balanced performance in thermochemistry and kinetics. Besides, as the LR-HF exchange contributions (see Eq. (77)) in SLC-PBE-D3, LC- $\omega$ PBE, and $\omega$ B97 are the same (with $\omega_{\mathrm{LR}}=0.40 \mathrm{bohr}^{-1}$ ), SLC-PBED3, LC- $\omega$ PBE, and $\omega$ B97 should have similar performance for the properties sensitive to the tail contributions. In addition, the SR-HF exchange contribution (see Eq. (6) ) in SLCPBE-D3 is significant only in the region of $r_{12} \lesssim 1 / \omega_{\mathrm{SR}}=0.5$ bohr (i.e., the same as the SR region identified by Chai and Head-Gordon [115]), and hence, should be responsible only for 
the properties sensitive to the core contributions. By contrast, for SLC-LDA-D3, a larger fraction of HF exchange is needed to reduce the severe error associated with the underlying LDA. Interestingly, the HF exchange operators adopted in SLC-LDA-D3 and SLC-PBED3 look upside down, when compared with those adopted in the MR hybrid functionals developed by Henderson et al. for different purposes [129].

As the HF exchange operator adopted in SLC-PBE-D3 has been optimized, the same HF exchange operator is adopted in SLC-B97-D3 without further optimization. However, the remaining D3 parameters $\left(s_{r, 6}\right.$ and $\left.s_{r, 8}\right)$ and B97 linear expansion coefficients $\left(c_{x \sigma, i}, c_{c \sigma \sigma, i}\right.$, and $\left.c_{c \alpha \beta, i}\right)$ of SLC-B97-D3 are determined self-consistently by a least-squares fitting procedure described in Ref. [79] (using the same training set), with the SLC-PBE-D3 orbitals being the initial guess orbitals. During the parameter optimization, as the statistical errors of the training set for SLC-B97-D3 are not significantly improved for $m>4$, the functional expansions adopted in SLC-B97-D3 are truncated at $m=4$. We summarize the optimized parameters of SLC-B97-D3 in Table I.

In the following sections, the overall performance of SLC-LDA-D3, SLC-PBE-D3, and SLC-B97-D3 will be compared with a popular semilocal functional:

\section{- $\mathrm{PBE}[17]$,}

and several widely used LC hybrid functionals:

- LC- $\omega$ PBE [77],

- $\omega$ B97 [79],

- $\omega \mathrm{B} 97 \mathrm{X}[\mathrm{79}]$,

- $\omega$ B97X-D [80],

- $\omega$ B97X-D3 [85]

on the training set and various test sets [130].

\section{RESULTS FOR THE TRAINING SET}

All calculations are performed with a development version of Q-Chem 4.3 [131]. Spinrestricted theory is used for singlet states and spin-unrestricted theory for others, unless 
noted otherwise. For the interaction energies of the weakly bound systems, the counterpoise correction [132] is employed to reduce the basis set superposition error (BSSE).

Results for the training set are computed using the $6-311++\mathrm{G}(3 \mathrm{df}, 3 \mathrm{pd})$ basis set with the fine grid $\operatorname{EML}(75,302)$, consisting of 75 Euler-Maclaurin radial grid points [133] and 302 Lebedev angular grid points [134]. The error for each entry is defined as error $=$ theoretical value - reference value. The notation adopted for characterizing statistical errors is as follows: mean signed errors (MSEs), mean absolute errors (MAEs), and root-mean-square (rms) errors.

As shown in Table II, SLC-PBE-D3 consistently outperforms PBE and LC- $\omega$ PBE for the AEs of the G3/99 set and noncovalent interactions of the S22 set, reflecting the effect of the improved HF exchange operator and dispersion correction, respectively. To provide the fairest comparison to SLC-PBE-D3, the performance of LC- $\omega$ PBE-D3 (i.e., LC- $\omega$ PBE with the D3 dispersion correction) [91] is also examined here. While LC- $\omega$ PBE-D3 performs similarly to SLC-PBE-D3 for the S22 set due to the inclusion of dispersion correction, LC$\omega$ PBE-D3 performs considerably worse than SLC-PBE-D3 for the G3/99 set.

Owing to its flexible functional forms, SLC-B97-D3 generally outperforms SLC-PBE-D3, and significantly outperforms SLC-LDA-D3 on the training set. Besides, as the fractions of HF exchange adopted in SLC-B97-D3 and $\omega$ B97 are similar in the MR region, SLCB97-D3 performs similarly to $\omega$ B97 for thermochemistry and kinetics, implying that the SR-HF exchange contribution in SLC-B97-D3 does not degrade its performance for normal chemistry. However, as mentioned previously, the HF exchange operator of SLC-PBE-D3 is adopted in SLC-B97-D3 (i.e., without further optimization), though the D3 parameters and B97 linear expansion coefficients of SLC-B97-D3 are optimized on the training set. Therefore, SLC-B97-D3 performs slightly worse than wB97X-D3 (where the HF exchange operator, D3 parameters, and B97 linear expansion coefficients were fully optimized on the same training set). All the dispersion-corrected functionals perform reasonably well for the noncovalent interactions of the S22 set.

\section{RESULTS FOR THE TEST SETS}

To examine how SLC-LDA-D3, SLC-PBE-D3, and SLC-B97-D3 perform outside the training set, we also assess their performance on a wide variety of test sets, including 
- the 23 core ionization energies of 14 molecules [135],

- the 38 core excitation energies of 13 molecules [114],

- the 66 noncovalent interactions of the S66 set [136],

- four dissociation energy curves of symmetric radical cations [22],

- the 113 AEs of the AE113 database [52, 84],

- the 131 vertical IPs of the IP131 database [84],

- the 131 vertical EAs of the EA131 database [52, 84],

- the 131 fundamental gaps of the FG131 database [52, 84],

- the 19 valence and 23 Rydberg excitation energies of five molecules [137],

- one long-range CT excitation energy curve of two well-separated molecules [55, 138].

As will be discussed later, each vertical IP can be computed in two different ways, each vertical EA can be computed in three different ways, and each fundamental gap can be computed in three different ways. Consequently, there are in total 1335 pieces of data in the test sets, which are larger and more diverse than the training set.

\section{A. Core ionization energies}

To assess the accuracy of the density functionals on core ionization energies, the 23 core ionization energies of 14 molecules are collected from Ref. [135], where the atoms at which the $1 s$ electrons are ionized are all first-row elements. As discussed by Baerends and coworkers [139], the ionization energies for all the occupied orbitals can be well approximated by the minus orbital energies, when the exact (or highly accurate) XC potential is adopted. Therefore, in this work, the core ionization energy of a molecule is calculated as the minus core orbital energy of the molecule, using the $6-311++\mathrm{G}(3 \mathrm{df}, 3 \mathrm{pd})$ basis set and $\mathrm{EML}(75,302)$ grid.

As shown in Table ஹI, PBE performs worst for the core ionization energies, while LC$\omega \mathrm{PBE}$ and $\omega \mathrm{B} 97$ only have minor improvement due to the vanishingly small fraction of HF 
exchange at small interelectronic distances. Besides, $\omega$ B97X, $\omega$ B97X-D, and $\omega$ B97X-D3, which include a small fraction of SR-HF exchange, perform slightly better than LC- $\omega$ PBE and $\omega$ B97. Among the functionals examined on the core ionization energies, SLC-B97D3 ranks first, while SLC-PBE-D3 and SLC-LDA-D3 rank second and third, respectively. Overall, the SLC hybrid functionals are comparable in performance, and are much more accurate than PBE, LC- $\omega$ PBE, the $\omega$ B97 series, and possibly, other LC hybrid functionals employing a small fraction of HF exchange in the SR region, reflecting that a very large fraction of $\mathrm{HF}$ exchange in the SR region is indeed essential for an accurate description of core ionization energies. While the relativistic corrections are not considered here, our comments remain the same for the core ionization energies with the relativistic corrections [130].

\section{B. Core excitation energies}

To examine if our SLC hybrid functionals also improve upon the other functionals for core excitation energies, we take the 38 core excitation energies of 13 molecules from Ref. [114], containing a total of 15 core $\rightarrow$ valence and 23 core $\rightarrow$ Rydberg excitation energies for the firstand second-row nuclei (from the $1 s$ core orbitals). In conventional TDDFT, the calculations of core excited states can be prohibitively expensive, owing to the large number of roots required to obtain the high energy core excited states. Following Besley et al. [114], we perform TDDFT calculations using the Tamm-Dancoff approximation (TDA) [137] within a reduced single excitation space (which includes only excitations from the core orbitals of interest) [140], to reduce the computational costs of core excitation energies. The calculations are performed with the $6-311(2+, 2+) \mathrm{G}^{* *}$ basis set and $\operatorname{EML}(100,302)$ grid.

For the core excitation energies (see Table IV), PBE, LC- $\omega$ PBE, and $\omega$ B97 perform very poorly, while $\omega \mathrm{B} 97 \mathrm{X}, \omega \mathrm{B} 97 \mathrm{X}-\mathrm{D}$, and $\omega \mathrm{B} 97 \mathrm{X}-\mathrm{D} 3$ only have minor improvement, due to the small fraction of HF exchange in the SR region. By contrast, SLC-PBE-D3 and SLC-B97D3 perform comparably, slightly improve upon SLC-LDA-D3, and significantly outperform PBE, LC- $\omega$ PBE, the $\omega$ B97 series, and perhaps, other LC hybrid functionals adopting a small fraction of $\mathrm{HF}$ exchange in the SR region. For the core excitation energies, the statistical errors associated with SLC-PBE-D3 and SLC-B97-D3 are about one order of magnitude smaller than those associated with PBE, LC- $\omega$ PBE, and the $\omega$ B97 series! Therefore, the 
inclusion of a very large fraction of HF exchange at small interelectronic distances is also important for accurately describing core excitation energies. While we do not consider the relativistic corrections here, our comments remain similar for the core excitation energies with the relativistic corrections [130].

\section{Noncovalent interactions}

For the noncovalent interactions of the S66 set [136], the performance of the functionals is evaluated using the $6-311++\mathrm{G}(3 \mathrm{df}, 3 \mathrm{pd})$ basis set and $\operatorname{EML}(99,590)$ grid, and the counterpoise correction [132] is adopted to reduce the BSSE. As shown in Table V], PBE and LC- $\omega$ PBE perform very poorly for the noncovalent interactions of the S66 set, due to the lack of a proper description of middle- and long-range dynamical correlation effects, while all the dispersion-corrected functionals perform reasonably well.

\section{Dissociation of symmetric radical cations}

Due to the pronounced SIEs associated with semilocal density functionals, unphysical fractional charge dissociation can happen, especially for symmetric charged radicals [22]. Here, the dissociation energy curves of $\mathrm{H}_{2}^{+}, \mathrm{He}_{2}^{+}, \mathrm{Ne}_{2}^{+}$, and $\mathrm{Ar}_{2}^{+}$are calculated using the $6-311++\mathrm{G}(3 \mathrm{df}, 3 \mathrm{pd})$ basis set and $\operatorname{EML}(75,302)$ grid to examine the performance of the functionals upon the SIE problems. The results are compared with the $\mathrm{H}_{2}^{+}$curve calculated using the HF theory (exact for any one-electron system) and the $\mathrm{He}_{2}^{+}, \mathrm{Ne}_{2}^{+}$, and $\mathrm{Ar}_{2}^{+}$curves calculated using the highly accurate $\operatorname{CCSD}(\mathrm{T})$ theory (coupled-cluster theory with iterative singles and doubles and perturbative treatment of triple substitutions) [141].

As shown in Figures 2 to 5, unphysical barriers indeed appear in the PBE dissociation curves, owing to the significant SIEs of PBE. By contrast, the LC and SLC hybrid functionals greatly reduce (or even remove) the unphysical barriers of the dissociation curves, due to the inclusion of $100 \%$ LR-HF exchange. SLC-LDA-D3, adopting the largest $\omega_{\mathrm{LR}}\left(0.45 \mathrm{bohr}^{-1}\right)$, performs best, followed by SLC-PBE-D3, SLC-B97-D3, LC- $\omega$ PBE, and $\omega$ B97, adopting the second largest $\omega_{\mathrm{LR}}\left(0.40 \mathrm{bohr}^{-1}\right)$. 


\section{E. Atomization energies}

Recently, we have developed the IP131, EA131, and FG131 databases [52, 84], consisting of accurate reference values for the 131 vertical IPs, 131 vertical EAs, and 131 fundamental gaps, respectively, of 18 atoms and 113 molecules at their experimental geometries. In addition, we have developed the AE113 database [52], which contains accurate reference values for the atomization energies of 113 molecules in the IP131 database. Here, we examine the performance of the functionals on the AE113, IP131, EA131, and FG131 databases, using the $6-311++\mathrm{G}(3 \mathrm{df}, 3 \mathrm{pd})$ basis set and $\operatorname{EML}(75,302)$ grid.

As shown in Table VI, owing to their flexible functional forms, SLC-B97-D3 and the $\omega$ B97 series are comparable in performance, more accurate than SLC-PBE-D3, and much more accurate than PBE, LC- $\omega$ PBE, and SLC-LDA-D3. Interestingly, SLC-PBE-D3 performs better than LC- $\omega \mathrm{PBE}$, possibly due to the noticeable deviation of their HF exchange operators in the region of 0.5 bohr $\lesssim r_{12} \lesssim 0.8$ bohr (where the fractions of HF exchange adopted in SLC-PBE-D3, $\omega$ B97X-D, and $\omega$ B97X-D3 are very similar!).

\section{F. Vertical ionization potentials}

The vertical IP of a molecule (containing $N$ electrons) is defined as

$$
\operatorname{IP}(1)=E_{\text {total }}(N-1)-E_{\text {total }}(N),
$$

where $E_{\text {total }}(N)$ is the total energy of the $N$-electron system. For the exact KS-DFT, the vertical IP of a molecule is the same as the minus HOMO energy of the molecule [33 38],

$$
\operatorname{IP}(2)=-\epsilon_{\text {HOMO }}(N) .
$$

However, for an approximate XC density functional in KS-DFT, the computed IP(1) and $\mathrm{IP}(2)$ values may be different, showing the accuracy of the predicted total energies and HOMO energies, respectively.

Here, we examine the accuracy of the functionals on the IP131 database [84], and summarize our results in Table VII. For IP(1), the $\omega$ B97 series, SLC-PBE-D3, and SLC-B97-D3 are comparable in performance, outperforming the other functionals. For IP(2), LC- $\omega$ PBE, $\omega B 97$, SLC-PBE-D3, and SLC-B97-D3, which adopt $\omega_{\mathrm{LR}}=0.40 \mathrm{bohr}^{-1}$, perform comparably, and outperform the other functionals. By contrast, PBE severely underestimates IP(2), 
due to the incorrect XC potential asymptote. For the IP(1) and IP(2) values, SLC-PBE-D3 and SLC-B97-D3 achieve the best performance, followed closely by $\omega$ B97.

\section{G. Vertical electron affinities}

The vertical EA of a molecule is defined as

$$
\operatorname{EA}(1)=E_{\text {total }}(N)-E_{\text {total }}(N+1) .
$$

By comparing Eq. (23) with Eq. (25), the vertical EA of a molecule is identical to the vertical IP of the corresponding anion, which is, for the exact KS-DFT, the minus HOMO energy of the anion,

$$
\mathrm{EA}(2)=-\epsilon_{\mathrm{HOMO}}(N+1) .
$$

In addition, the vertical EA of a molecule is traditionally approximated by the minus lowest unoccupied molecular orbital (LUMO) energy of the molecule,

$$
\operatorname{EA}(3)=-\epsilon_{\text {LUMO }}(N)
$$

Nonetheless, even for the exact KS-DFT, there is a fundamental difference between EA(3) and $\mathrm{EA}(2)$, owing to the derivative discontinuity $\Delta_{x c}[34,38,142-146]$ of $E_{x c}[\rho]$ : $\mathrm{EA}(3)-$ $\operatorname{EA}(2)=\epsilon_{\text {HOMO }}(N+1)-\epsilon_{\text {LUMO }}(N)=\Delta_{x c}$. Hybrid density functionals, which belong to the generalized Kohn-Sham (GKS) method [147] (not pure KS-DFT), effectively capture a fraction of $\Delta_{x c}$ of $E_{x c}[\rho]$ in KS-DFT. A recent study has found that the difference between $\epsilon_{\mathrm{HOMO}}(N+1)$ and $\epsilon_{\mathrm{LUMO}}(N)$ is small for LC hybrid functionals [148]. Therefore, $\mathrm{EA}(3)$ is expected to be close to EA(2) (i.e., the true vertical EA) for LC hybrid functionals.

Here, the accuracy of the functionals on the EA131 database [52, 84] is investigated. As shown in Table VIII, all the functionals perform comparably for EA(1). However, for the $\mathrm{EA}(2)$ and $\mathrm{EA}(3)$ values, the LC and SLC hybrid functionals perform much better than PBE, showing the importance of LR-HF exchange in frontier orbital energies. Note that PBE significantly underestimates EA(2), due to the incorrect asymptotic behavior of the XC potential. However, for EA(3), there is a fortuitous cancellation of errors in the vertical EA calculated using $\mathrm{PBE}$, as $\epsilon_{\mathrm{LUMO}}(N)$ is incorrectly upshifted due to the incorrect $\mathrm{PBE} \mathrm{XC}$ potential asymptote, effectively capturing a fraction of $\Delta_{x c}$ [52]. Similar to the LC hybrid functionals, $\Delta_{x c}$ is also found to be close to zero for the SLC hybrid functionals, which can 
be attributed to the LR-HF exchange adopted in the LC and SLC hybrid functionals. For the EA(1), EA(2), and EA(3) values, SLC-PBE-D3 performs best, followed by SLC-B97-D3.

\section{H. Fundamental gaps}

The fundamental gap $E_{g}$ of a molecule is the difference between the vertical IP and EA of the molecule, i.e., $E_{g}=$ IP - EA. As mentioned previously, there are various ways of calculating the vertical IP and EA in KS-DFT. Here, we adopt the following three popular ways to calculate $E_{g}$ :

$$
\begin{aligned}
& E_{g}(1)=\operatorname{IP}(1)-\mathrm{EA}(1)=E_{\text {total }}(N-1)+E_{\text {total }}(N+1)-2 E_{\text {total }}(N) \\
& E_{g}(2)=\operatorname{IP}(2)-\mathrm{EA}(2)=\epsilon_{\mathrm{HOMO}}(N+1)-\epsilon_{\mathrm{HOMO}}(N) \\
& E_{g}(3)=\operatorname{IP}(2)-\mathrm{EA}(3)=\epsilon_{\mathrm{LUMO}}(N)-\epsilon_{\mathrm{HOMO}}(N)
\end{aligned}
$$

Note that $E_{g}(3)$ is the HOMO-LUMO gap in KS-DFT (i.e., the KS gap). For the exact KS-DFT, both $E_{g}(1)$ and $E_{g}(2)$ lead to the exact fundamental gap, but there is a distinct difference between $E_{g}(2)$ and $E_{g}(3)$ (i.e., the energy-gap problem), due to the $\Delta_{x c}$ of $E_{x c}[\rho]$ : $E_{g}(2)-E_{g}(3)=\mathrm{EA}(3)-\operatorname{EA}(2)=\Delta_{x c}$. For the LC and SLC hybrid functionals, as $\mathrm{EA}(3)$ is close to $\operatorname{EA}(2), E_{g}(3)$ should be close to $E_{g}(2)$ (i.e., the true fundamental gap).

Here, we assess the accuracy of the functionals on the FG131 database [52, 84]. As shown in Table IX, for $E_{g}(1)$, the $\omega$ B97 series, SLC-PBE-D3, and SLC-B97-D3 are comparable in performance, outperforming the other functionals. For $E_{g}(2)$, SLC-PBE-D3 and SLC-B97D3 perform best, followed closely by $\omega$ B97. For $E_{g}(3)$, PBE performs worst due to the lack of $\Delta_{x c}$, while SLC-PBE-D3, SLC-B97-D3, and $\omega$ B97 perform well for the energy-gap problems

here. For the $E_{g}(1), E_{g}(2)$, and $E_{g}(3)$ values, SLC-PBE-D3 ranks first, SLC-B97-D3 ranks second, and $\omega \mathrm{B} 97$ ranks third.

\section{Valence and Rydberg excitation energies}

To examine the performance of the functionals on valence and Rydberg excitation energies, TDDFT calculations are performed on five molecules, involving nitrogen gas $\left(\mathrm{N}_{2}\right)$, carbon monoxide $(\mathrm{CO})$, water $\left(\mathrm{H}_{2} \mathrm{O}\right)$, ethylene $\left(\mathrm{C}_{2} \mathrm{H}_{4}\right)$, and formaldehyde $\left(\mathrm{CH}_{2} \mathrm{O}\right)$, using the 6-311 $(2+, 2+) \mathrm{G}^{* *}$ basis set and $\operatorname{EML}(99,590)$ grid. The experimental excitation energies are 
taken from Ref. [137].

As shown in Table X, all the functionals perform reasonably well for the valence excitation energies. However, PBE severely underestimates the Rydberg excitation energies due to the incorrect $\mathrm{XC}$ potential asymptote, while the LC and SLC hybrid functionals perform reasonably well here.

\section{J. Long-range charge-transfer excitation energies}

Dreuw et al. have shown that the correct CT excitation energy from the HOMO of a donor to the LUMO of an acceptor should possess the following asymptote [55]:

$$
\omega_{\mathrm{CT}}(R \rightarrow \infty) \approx \mathrm{IP}_{\mathrm{D}}-\mathrm{EA}_{\mathrm{A}}-1 / R
$$

where $\mathrm{IP}_{\mathrm{D}}$ is the IP of the donor, $\mathrm{EA}_{\mathrm{A}}$ is the $\mathrm{EA}$ of the acceptor, and $R$ is the intermolecular distance.

Following Dreuw et al., we perform TDDFT calculations for the lowest CT excitation energy between ethylene and tetrafluoroethylene with a separation of $R$, using the $6-31 \mathrm{G}^{*}$ basis set and $\operatorname{EML}(99,590)$ grid. High-level ab initio results obtained with the symmetryadapted-cluster configuration-interaction (SAC-CI) method are taken from Tawada et al. for comparison [138]. Unsurprisingly, the LC and SLC hybrid functionals, which retain $100 \%$ LR-HF exchange, yield the correct $(-1 / R)$ asymptote in the calculated $\omega_{\mathrm{CT}}(R)$ (see Figure 6). Nevertheless, as shown in Figure 7, the long-range CT excitation energies are rather sensitive to the LR behavior of the HF exchange operator (i.e., $\omega_{\mathrm{LR}}$ ), and relatively insensitive to the SR behavior of the HF exchange operator (i.e., $\omega_{\mathrm{SR}}$ ). SLC-LDA-D3, which adopts the largest $\omega_{\mathrm{LR}}\left(0.45 \mathrm{bohr}^{-1}\right)$, performs best, followed by SLC-B97-D3, SLC-PBE-D3, LC- $\omega$ PBE, and $\omega$ B97, which adopt the second largest $\omega_{\mathrm{LR}}\left(0.40 \mathrm{bohr}^{-1}\right)$.

\section{CONCLUSIONS}

In summary, we have proposed the SLC hybrid scheme employing $100 \% \mathrm{HF}$ exchange at both zero and infinite interelectronic distances, wherein three SLC hybrid density functionals with the D3 dispersion corrections have been developed. Owing to a very large fraction of HF exchange in the SR region, our SLC-LDA-D3, SLC-PBE-D3, and SLC-B97-D3 functionals yield much more accurate core ionization and excitation energies than LC- $\omega \mathrm{PBE}$ and the 
$\omega \mathrm{B} 97$ series. Besides, due to a similar fraction of HF exchange in the MR and LR regions, SLC-PBE-D3 and SLC-B97-D3 are generally comparable or superior to LC- $\omega$ PBE and the $\omega$ B97 series, respectively, in performance, for many other test sets, such as dissociation of symmetric radical cations, atomization energies, vertical IPs, vertical EAs, fundamental gaps, and valence, Rydberg, and long-range CT excitation energies. For noncovalent interactions, SLC-LDA-D3, SLC-PBE-D3, SLC-B97-D3, and the other dispersion-corrected functionals perform reasonably well. Relative to $\omega$ B97X-D, SLC-B97-D3 provides significant improvement for core ionization and excitation energies and noticeable improvement for the SIE, asymptote, energy-gap, and CT problems, while performing similarly for thermochemistry, kinetics, and noncovalent interactions.

By construction, the SLC hybrid scheme can perform reasonably well for the properties sensitive to the SR (e.g., core ionization and excitation energies), MR (e.g., thermochemistry and kinetics), and LR (e.g., the SIE, asymptote, energy-gap, and CT problems) behavior of the HF exchange operator. For the properties insensitive to the HF exchange operator (e.g., noncovalent interactions), the SLC hybrid scheme does not necessarily yield good accuracy. Nevertheless, to provide an accurate description of noncovalent interactions, the SLC hybrid scheme can be combined with the DFT-D schemes, the double-hybrid schemes, and fully nonlocal correlation density functionals for van der Waals interactions. Alternatively, the SLC hybrid scheme can also be extended to the recently developed MGGAs with mediumrange correlation relevant for noncovalent interactions (e.g., the MGGA_MS family [149 151] and SCAN [19]), provided that the corresponding MGGA exchange functionals of the MR operator (see Eq. (묘) ) are devised.

\section{ACKNOWLEDGMENTS}

This work was supported by the Ministry of Science and Technology of Taiwan (Grant No. MOST104-2628-M-002-011-MY3), National Taiwan University (Grant No. NTU-CDP105R7818), the Center for Quantum Science and Engineering at NTU (Subproject Nos.: NTU-ERP-105R891401 and NTU-ERP-105R891403), and the National Center for Theoret- 
ical Sciences of Taiwan.

[1] P. Hohenberg and W. Kohn, Phys. Rev. 136, B864 (1964).

[2] W. Kohn and L. J. Sham, Phys. Rev. 140, A1133 (1965).

[3] R. G. Parr and W. Yang, Density-Functional Theory of Atoms and Molecules (Oxford University, New York, 1989).

[4] S. Kümmel and L. Kronik, Rev. Mod. Phys. 80, 3 (2008).

[5] A. J. Cohen, P. Mori-Sánchez, and W. Yang, Chem. Rev. 112, 289 (2011).

[6] E. Engel and R. M. Dreizler, Density Functional Theory: An Advanced Course (Springer, Heidelberg, 2011).

[7] E. Runge and E. K. U. Gross, Phys. Rev. Lett. 52, 997 (1984).

[8] M. E. Casida, Recent Advances in Density Functional Methods, Part I (World Scientific, Singapore, 1995).

[9] E. K. U. Gross, J. F. Dobson, and M. Petersilka, in Density Functional Theory II (Springer, New York, 1996), vol. 181, pp. 81-172.

[10] M. Petersilka, U. J. Gossmann, and E. K. U. Gross, Phys. Rev. Lett. 76, 1212 (1996).

[11] M. E. Casida, C. Jamorski, K. C. Casida, and D. R. Salahub, J. Chem. Phys. 108, 4439 (1998).

[12] G. Onida, L. Reining, and A. Rubio, Rev. Mod. Phys. 74, 601 (2002).

[13] M. A. L. Marques and E. K. U. Gross, Annu. Rev. Phys. Chem. 55, 427 (2004).

[14] C. A. Ullrich, Time-Dependent Density-Functional Theory: Concepts and Applications (Oxford University, New York, 2012).

[15] P. A. M. Dirac, Proc. Cambridge Philos. Soc. 26, 376 (1930).

[16] J. P. Perdew and Y. Wang, Phys. Rev. B 45, 13244 (1992).

[17] J. P. Perdew, K. Burke, and M. Ernzerhof, Phys. Rev. Lett. 77, 3865 (1996).

[18] J. Tao, J. P. Perdew, V. N. Staroverov, and G. E. Scuseria, Phys. Rev. Lett. 91, 146401 (2003).

[19] J. Sun, A. Ruzsinszky, and J. P. Perdew, Phys. Rev. Lett. 115, 036402 (2015).

[20] J. P. Perdew, A. Ruzsinszky, J. Tao, V. N. Staroverov, G. E. Scuseria, G. I. Csonka, J. Chem. Phys. 123, 062201 (2005). 
[21] A. J. Cohen, P. Mori-Sánchez, and W. Yang, Science 321, 792 (2008).

[22] T. Bally and G. N. Sastry, J. Phys. Chem. A 101, 7923 (1997); B. Braïda, P. C. Hiberty, and A. Savin, J. Phys. Chem. A 102, 7872 (1998); M. Grüning, O. V. Gritsenko, S. J. A. van Gisbergen, and E. J. Baerends, J. Phys. Chem. A 105, 9211 (2001); D. J. Tozer, N. C. Handy, and A. J. Cohen, Chem. Phys. Lett. 382, 203 (2003); M. Lundberg and P. E. M. Siegbahn, J. Chem. Phys. 122, 224103 (2005); P. Mori-Sánchez, A. J. Cohen, and W. Yang, J. Chem. Phys. 125, 201102 (2006); A. D. Dutoi and M. Head-Gordon, Chem. Phys. Lett. 422, 230 (2006); A. Ruzsinszky, J. P. Perdew, G. I. Csonka, O. A. Vydrov, and G. E. Scuseria, J. Chem. Phys. 126, 104102 (2007).

[23] J. F. Dobson, K. McLennan, A. Rubio, J. Wang, T. Gould, H. M. Le, and B. P. Dinte, Aust. J. Chem. 54, 513 (2001).

[24] S. Grimme, A. Hansen, J. G. Brandenburg, and C. Bannwarth, Chem. Rev. 116, 5105 (2016).

[25] Y.-T. Chen, K. Hui, and J.-D. Chai, Phys. Chem. Chem. Phys. 18, 3011 (2016).

[26] G. Gryn'ova, M. L. Coote, and C. Corminboeuf, WIREs Comput. Mol. Sci. 5, 440 (2015).

[27] J.-D. Chai, J. Chem. Phys. 136, 154104 (2012).

[28] J.-D. Chai, J. Chem. Phys. 140, 18A521 (2014).

[29] C.-S. Wu and J.-D. Chai, J. Chem. Theory Comput. 11, 2003 (2015).

[30] C.-N. Yeh and J.-D. Chai, Sci. Rep. 6, 30562 (2016).

[31] S. Seenithurai and J.-D. Chai, Sci. Rep. 6, 33081 (2016).

[32] C.-S. Wu, P.-Y. Lee, and J.-D. Chai, e-print arXiv:1607.04900.

[33] J. F. Janak, Phys. Rev. B 18, 7165 (1978).

[34] J. P. Perdew, R. G. Parr, M. Levy, and J. L. Balduz, Jr., Phys. Rev. Lett. 49, 1691 (1982).

[35] M. Levy, J. P. Perdew, and V. Sahni, Phys. Rev. A 30, 2745 (1984).

[36] C.-O. Almbladh and U. von Barth, Phys. Rev. B 31, 3231 (1985).

[37] J. P. Perdew and M. Levy, Phys. Rev. B 56, 16021 (1997).

[38] M. E. Casida, Phys. Rev. B 59, 4694 (1999).

[39] M. E. Casida and D. R. Salahub, J. Chem. Phys. 113, 8918 (2000).

[40] N. N. Matsuzawa, A. Ishitani, D. A. Dixon, and T. Uda, J. Phys. Chem. A 105, 4953 (2001).

[41] M. J. G. Peach, P. Benfield, T. Helgaker, and D. J. Tozer, J. Chem. Phys. 128, 044118 (2008).

[42] C.-R. Pan, P.-T. Fang, and J.-D. Chai, Phys. Rev. A 87, 052510 (2013). 
[43] R. Armiento and S. Kümmel, Phys. Rev. Lett. 111, 036402 (2013).

[44] J. Carmona-Espíndola, J. L. Gázquez, A. Vela, and S. B. Trickey, J. Chem. Phys. 142, 054105 (2015).

[45] S. L. Li and D. G. Truhlar, J. Chem. Theory Comput. 11, 3123 (2015).

[46] H.-L. Sun, W.-T. Peng, and J.-D. Chai, RSC Adv. 6, 33318 (2016).

[47] R. van Leeuwen and E. J. Baerends, Phys. Rev. A 49, 2421 (1994).

[48] P. R. T. Schipper, O. V. Gritsenko, S. J. A. van Gisbergen, and E. J. Baerends, J. Chem. Phys. 112, 1344 (2000).

[49] D. J. Tozer, J. Chem. Phys. 112, 3507 (2000).

[50] X. Andrade and A. Aspuru-Guzik, Phys. Rev. Lett. 107, 183002 (2011).

[51] A. P. Gaiduk, D. S. Firaha, and V. N. Staroverov, Phys. Rev. Lett. 108, 253005 (2012).

[52] C.-W. Tsai, Y.-C. Su, G.-D. Li, and J.-D. Chai, Phys. Chem. Chem. Phys. 15, 8352 (2013).

[53] D. J. Tozer, R. D. Amos, N. C. Handy, B. O. Roos, and L. Serrano-Andres, Mol. Phys. 97, 859 (1999).

[54] D. J. Tozer, J. Chem. Phys. 119, 12697 (2003).

[55] A. Dreuw, J. L. Weisman, and M. Head-Gordon, J. Chem. Phys. 119, 2943 (2003).

[56] A. Dreuw and M. Head-Gordon, J. Am. Chem. Soc. 126, 4007 (2004).

[57] O. Gritsenko and E. J. Baerends, J. Chem. Phys. 121, 655 (2004).

[58] J. Neugebauer, O. Gritsenko, and E. J. Baerends, J. Chem. Phys. 124, 214102 (2006).

[59] B. M. Wong and J. G. Cordaro, J. Chem. Phys. 129, 214703 (2008).

[60] B. M. Wong, M. Piacenza, and F. D. Sala, Phys. Chem. Chem. Phys. 11, 4498 (2009).

[61] W.-T. Peng and J.-D. Chai, Phys. Chem. Chem. Phys. 16, 21564 (2014).

[62] M. Hellgren and E. K. U. Gross, Phys. Rev. A 85, 022514 (2012).

[63] A. D. Becke, J. Chem. Phys. 98, 1372 (1993).

[64] A. D. Becke, J. Chem. Phys. 98, 5648 (1993).

[65] P. J. Stephens, F. J. Devlin, C. F. Chabalowski, and M. J. Frisch, J. Phys. Chem. 98, 11623 (1994).

[66] J. P. Perdew, M. Ernzerhof, and K. Burke, J. Chem. Phys. 105, 9982 (1996).

[67] A. D. Becke, J. Chem. Phys. 107, 8554 (1997).

[68] C. Adamo and V. Barone, J. Chem. Phys. 110, 6158 (1999).

[69] M. Ernzerhof and G. E. Scuseria, J. Chem. Phys. 110, 5029 (1999). 
[70] A. D. Boese and J. M. L. Martin, J. Chem. Phys. 121, 3405 (2004).

[71] Y. Zhao and D. G. Truhlar, Theor. Chem. Acc. 120, 215 (2008).

[72] K. Hui and J.-D. Chai, J. Chem. Phys. 144, 044114 (2016).

[73] J.-C. Lee, J.-D. Chai, and S.-T. Lin, RSC Adv. 5, 101370 (2015).

[74] A. Savin, in Recent Developments and Applications of Modern Density Functional Theory, edited by J. M. Seminario (Elsevier, Amsterdam, 1996), pp. 327-357.

[75] H. Iikura, T. Tsuneda, T. Yanai, and K. Hirao, J. Chem. Phys. 115, 3540 (2001).

[76] T. Yanai, D. P. Tew, and N. C. Handy, Chem. Phys. Lett. 393, 51 (2004).

[77] O. A. Vydrov, J. Heyd, A. V. Krukau, and G. E. Scuseria, J. Chem. Phys. 125, 074106 (2006).

[78] E. Livshits and R. Baer, Phys. Chem. Chem. Phys. 9, 2932 (2007).

[79] J.-D. Chai and M. Head-Gordon, J. Chem. Phys. 128, 084106 (2008).

[80] J.-D. Chai and M. Head-Gordon, Phys. Chem. Chem. Phys. 10, 6615 (2008).

[81] M. A. Rohrdanz and J. M. Herbert, J. Chem. Phys. 129, 034107 (2008).

[82] J.-D. Chai and M. Head-Gordon, J. Chem. Phys. 131, 174105 (2009).

[83] R. Peverati and D. G. Truhlar, J. Phys. Chem. Lett. 2, 2810 (2011).

[84] Y.-S. Lin, C.-W. Tsai, G.-D. Li, and J.-D. Chai, J. Chem. Phys. 136, 154109 (2012).

[85] Y.-S. Lin, G.-D. Li, S.-P. Mao, and J.-D. Chai, J. Chem. Theory Comput. 9, 263 (2013).

[86] N. Mardirossian and M. Head-Gordon, Phys. Chem. Chem. Phys. 16, 9904 (2014).

[87] N. Mardirossian and M. Head-Gordon, J. Chem. Phys. 144, 214110 (2016).

[88] S. Grimme, J. Comput. Chem. 25, 1463 (2004).

[89] S. Grimme, J. Comput. Chem. 27, 1787 (2006).

[90] S. Grimme, J. Antony, S. Ehrlich, and H. Krieg, J. Chem. Phys. 132, 154104 (2010).

[91] L. Goerigk and S. Grimme, Phys. Chem. Chem. Phys. 13, 6670 (2011).

[92] L. A. Burns, Á.Vázquez-Mayagoitia, B. G. Sumpter, and C. D. Sherrill, J. Chem. Phys. 134, 084107 (2011).

[93] S. Grimme, J. Chem. Phys. 124, 034108 (2006).

[94] I. Y. Zhang, X. Xu, and W. A. Goddard III, Proc. Natl. Acad. Sci. U.S.A. 106, 4963 (2009).

[95] K. Sharkas, J. Toulouse, and A. Savin, J. Chem. Phys. 134, 064113 (2011).

[96] E. Brémond and C. Adamo, J. Chem. Phys. 135, 024106 (2011).

[97] J. Toulouse, K. Sharkas, E. Brémond, and C. Adamo, J. Chem. Phys. 135, 101102 (2011). 
[98] I. Y. Zhang, N. Q. Su, É. A. G. Brémond, C. Adamo, and X. Xu, J. Chem. Phys. 136, $174103(2012)$.

[99] J.-D. Chai and S.-P. Mao, Chem. Phys. Lett. 538, 121 (2012).

[100] S. M. O. Souvi, K. Sharkas, and J. Toulouse, J. Chem. Phys. 140, 084107 (2014).

[101] N. Q. Su and X. Xu, J. Chem. Phys. 140, 18A512 (2014).

[102] E. Brémond, J. C. Sancho-García, A. J. Pérez-Jiménez, and C. Adamo, J. Chem. Phys. 141, 031101 (2014).

[103] J. Kim and Y. Jung, J. Chem. Theory Comput. 11, 45 (2015).

[104] M. Alipour, Theor. Chem. Acc. 134, 87 (2015).

[105] É. Brémond, M. Savarese, Á. J. Pérez-Jiménez, J. C. Sancho-García, and C. Adamo, J. Phys. Chem. Lett. 6, 3540 (2015).

[106] M. Dion, H. Rydberg, E. Schröder, D. C. Langreth, and B. I. Lundqvist, Phys. Rev. Lett. 92, 246401 (2004).

[107] O. A. Vydrov and T. Van Voorhis, J. Chem. Phys. 133, 244103 (2010).

[108] W. Hujo and S. Grimme, J. Chem. Theory Comput. 7, 3866 (2011).

[109] H.-C. Li, J.-D. Chai, and M.-K. Tsai, Int. J. Quan. Chem. 114, 805 (2014).

[110] T. M. Maier, H. Bahmann, A. V. Arbuznikov, and M. Kaupp, J. Chem. Phys. 144, 074106 (2016).

[111] A. Nakata, Y. Imamura, T. Ostuka, and H. Nakai, J. Chem. Phys. 124, 094105 (2006).

[112] A. Nakata, Y. Imamura, and H. Nakai, J. Chem. Phys. 125, 064109 (2006).

[113] J.-W. Song, M. A. Watson, A. Nakata, and K. Hirao, J. Chem. Phys. 129, 184113 (2008).

[114] N. A. Besley, M. J. G. Peach, and D. J. Tozer, Phys. Chem. Chem. Phys. 11, 10350 (2009).

[115] J.-D. Chai and M. Head-Gordon, Chem. Phys. Lett. 467, 176 (2008).

[116] P. M. W. Gill and R. D. Adamson, Chem. Phys. Lett. 261, 105 (1996).

[117] J. Toulouse, F. Colonna, and A. Savin, Phys. Rev. A 70, 062505 (2004).

[118] J.-W. Song, S. Tokura, T. Sato, M. A. Watson, and K. Hirao, J. Chem. Phys. 127, 154109 (2007).

[119] A. D. Dutoi and M. Head-Gordon, J. Phys. Chem. A 112, 2110 (2008).

[120] J. A. Parkhill, J.-D. Chai, A. D. Dutoi, and M. Head-Gordon, Chem. Phys. Lett. 478, 283 (2009).

[121] P. M. W. Gill, R. D. Adamson, and J. A. Pople, Mol. Phys. 88, 1005 (1996). 
[122] T. M. Henderson, B. G. Janesko, and G. E. Scuseria, J. Chem. Phys. 128, 194105 (2008).

[123] H. Stoll, C. M. E. Pavlidou, and H. Preuss, Theor. Chim. Acta 49, 143 (1978); H. Stoll, E. Golka, and H. Preuss, Theor. Chim. Acta 55, 29 (1980).

[124] L. A. Curtiss, K. Raghavachari, P. C. Redfern, and J. A. Pople, J. Chem. Phys. 106, 1063 (1997); L. A. Curtiss, P. C. Redfern, K. Raghavachari, and J. A. Pople, J. Chem. Phys. 109, 42 (1998); L. A. Curtiss, K. Raghavachari, P. C. Redfern, and J. A. Pople, J. Chem. Phys. 112, 7374 (2000).

[125] J. A. Pople, M. Head-Gordon, D. J. Fox, K. Raghavachari, and L. A. Curtiss, J. Chem. Phys. 90, 5622 (1989).

[126] Y. Zhao, B. J. Lynch, and D. G. Truhlar, J. Phys. Chem. A 108, 2715 (2004); Y. Zhao, N. González-García, and D. G. Truhlar, J. Phys. Chem. A 109, 2012 (2005); 110, 4942(E) (2006).

[127] P. Jurečka, J. Šponer, J. Černý, and P. Hobza, Phys. Chem. Chem. Phys. 8, 1985 (2006).

[128] M. S. Marshall, L. A. Burns, and C. D. Sherrill, J. Chem. Phys. 135, 194102 (2011).

[129] T. M. Henderson, A. F. Izmaylov, G. E. Scuseria, and A. Savin, J. Chem. Phys. 127, 221103 (2007)

[130] See supplementary material at (a link to be inserted) for further numerical results.

[131] Y. Shao, Z. Gan, E. Epifanovsky, A. T. B. Gilbert, M. Wormit, J. Kussmann, A. W. Lange, A. Behn, J. Deng, X. Feng, D. Ghosh, M. Goldey, P. R. Horn, L. D. Jacobson, I. Kaliman, R. Z. Khaliullin, T. Kuś, A. Landau, J. Liu, E. I. Proynov, Y. M. Rhee, R. M. Richard, M. A. Rohrdanz, R. P. Steele, E. J. Sundstrom, H. L. Woodcock III, P. M. Zimmerman, D. Zuev, B. Albrecht, E. Alguire, B. Austin, G. J. O. Beran, Y. A. Bernard, E. Berquist, K. Brandhorst, K. B. Bravaya, S. T. Brown, D. Casanova, C.-M. Chang, Y. Chen, S. H. Chien, K. D. Closser, D. L. Crittenden, M. Diedenhofen, R. A. DiStasio Jr., H. Do, A. D. Dutoi, R. G. Edgar, S. Fatehi, L. Fusti-Molnar, A. Ghysels, A. Golubeva-Zadorozhnaya, J. Gomes, M. W. D. Hanson-Heine, P. H. P. Harbach, A. W. Hauser, E. G. Hohenstein, Z. C. Holden, T.-C. Jagau, H. Ji, B. Kaduk, K. Khistyaev, J. Kim, J. Kim, R. A. King, P. Klunzinger, D. Kosenkov, T. Kowalczyk, C. M. Krauter, K. U. Lao, A. Laurent, K. V. Lawler, S. V. Levchenko, C. Y. Lin, F. Liu, E. Livshits, R. C. Lochan, A. Luenser, P. Manohar, S. F. Manzer, S.-P. Mao, N. Mardirossian, A. V. Marenich, S. A. Maurer, N. J. Mayhall, E. Neuscamman, C. M. Oana, R. Olivares-Amaya, D. P. O’Neill, J. A. Parkhill, T. M. Perrine, 
R. Peverati, A. Prociuk, D. R. Rehn, E. Rosta, N. J. Russ, S. M. Sharada, S. Sharma, D. W. Small, A. Sodt, T. Stein, D. Stück, Y.-C. Su, A. J. W. Thom, T. Tsuchimochi, V. Vanovschi, L. Vogt, O. Vydrov, T. Wang, M. A. Watson, J. Wenzel, A. White, C. F. Williams, J. Yang, S. Yeganeh, S. R. Yost, Z.-Q. You, I. Y. Zhang, X. Zhang, Y. Zhao, B. R. Brooks, G. K. L. Chan, D. M. Chipman, C. J. Cramer, W. A. Goddard III, M. S. Gordon, W. J. Hehre, A. Klamt, H. F. Schaefer III, M. W. Schmidt, C. D. Sherrill, D. G. Truhlar, A. Warshel, X. Xu, A. Aspuru-Guzik, R. Baer, A. T. Bell, N. A. Besley, J.-D. Chai, A. Dreuw, B. D. Dunietz, T. R. Furlani, S. R. Gwaltney, C.-P. Hsu, Y. Jung, J. Kong, D. S. Lambrecht, W. Z. Liang, C. Ochsenfeld, V. A. Rassolov, L. V. Slipchenko, J. E. Subotnik, T. Van Voorhis, J. M. Herbert, A. I. Krylov, P. M. W. Gill, and M. Head-Gordon, Mol. Phys. 113, 184 (2015).

[132] S. F. Boys and F. Bernardi, Mol. Phys. 19, 553 (1970).

[133] C. W. Murray, N. C. Handy, and G. J. Laming, Mol. Phys. 78, 997 (1993).

[134] V. I. Lebedev and D. N. Laikov, Dokl. Math. 59, 477 (1999).

[135] N. A. Besley, A. T. B. Gilbert, and P. M. W. Gill, J. Chem. Phys. 130, 124308 (2009).

[136] J. R̆ezác̆, K. E. Riley, and P. Hobza, J. Chem. Theory Comput. 7, 2427 (2011).

[137] S. Hirata and M. Head-Gordon, Chem. Phys. Lett. 314, 291 (1999).

[138] Y. Tawada, T. Tsuneda, S. Yanagisawa, T. Yanai, and K. Hirao, J. Chem. Phys. 120, 8425 (2004).

[139] D. P. Chong, O. V. Gritsenko, and E. J. Baerends, J. Chem. Phys. 116, 1760 (2002); O. V. Gritsenko and E. J. Baerends, J. Chem. Phys. 117, 9154 (2002); O. V. Gritsenko, B. Braïda, and E. J. Baerends, J. Chem. Phys. 119, 1937 (2003); O. V. Gritsenko and E. J. Baerends, J. Chem. Phys. 120, 8364 (2004).

[140] N. A. Besley, Chem. Phys. Lett. 390, 124 (2004).

[141] K. Raghavachari, G. W. Trucks, J. A. Pople, and M. Head-Gordon, Chem. Phys. Lett. 157, 479 (1989).

[142] J. P. Perdew and M. Levy, Phys. Rev. Lett. 51, 1884 (1983).

[143] L. J. Sham and M. Schlüter, Phys. Rev. Lett. 51, 1888 (1983).

[144] P. Mori-Sánchez, A. J. Cohen, and W. Yang, Phys. Rev. Lett. 102, 066403 (2009).

[145] X. Andrade and A. Aspuru-Guzik, Phys. Rev. Lett. 107, 183002 (2011).

[146] J.-D. Chai and P.-T. Chen, Phys. Rev. Lett. 110, 033002 (2013).

[147] A. Seidl, A. Görling, P. Vogl, J. A. Majewski, and M. Levy, Phys. Rev. B, 53, 3764 (1996). 
[148] T. Tsuneda, J.-W. Song, S. Suzuki, and K. Hirao, J. Chem. Phys. 133, 174101 (2010).

[149] J. Sun, B. Xiao, and A. Ruzsinszky, J. Chem. Phys. 137, 051101 (2012).

[150] J. Sun, R. Haunschild, B. Xiao, I. W. Bulik, G. E. Scuseria, and J. P. Perdew, J. Chem. Phys. 138, 044113 (2013).

[151] J. Sun, B. Xiao, Y. Fang, R. Haunschild, P. Hao, A. Ruzsinszky, G. I. Csonka, G. E. Scuseria, and J. P. Perdew, Phys. Rev. Lett. 111, 106401 (2013). 


\section{FIGURES}

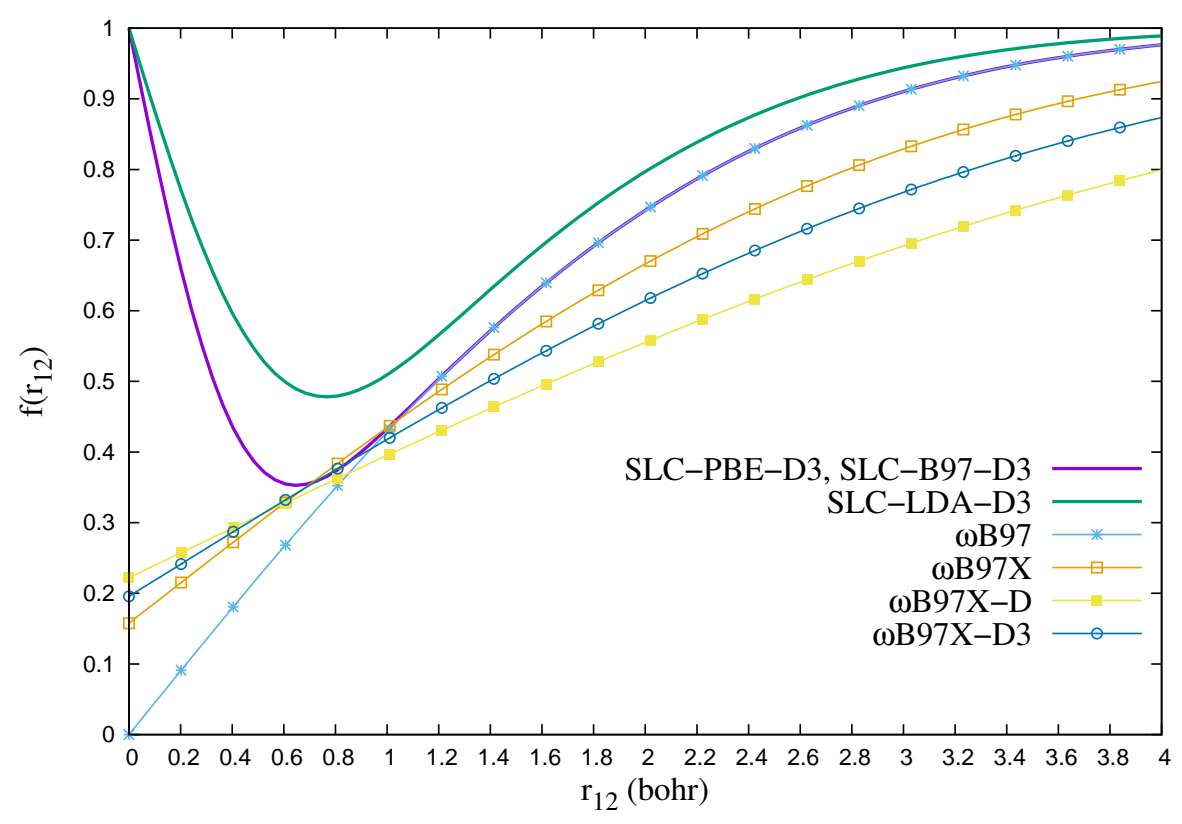

FIG. 1. Fraction of HF exchange $f\left(r_{12}\right)$ as a function of the interelectronic distance $r_{12}$, for SLCLDA-D3, SLC-PBE-D3, SLC-B97-D3, and the $\omega$ B97 series.

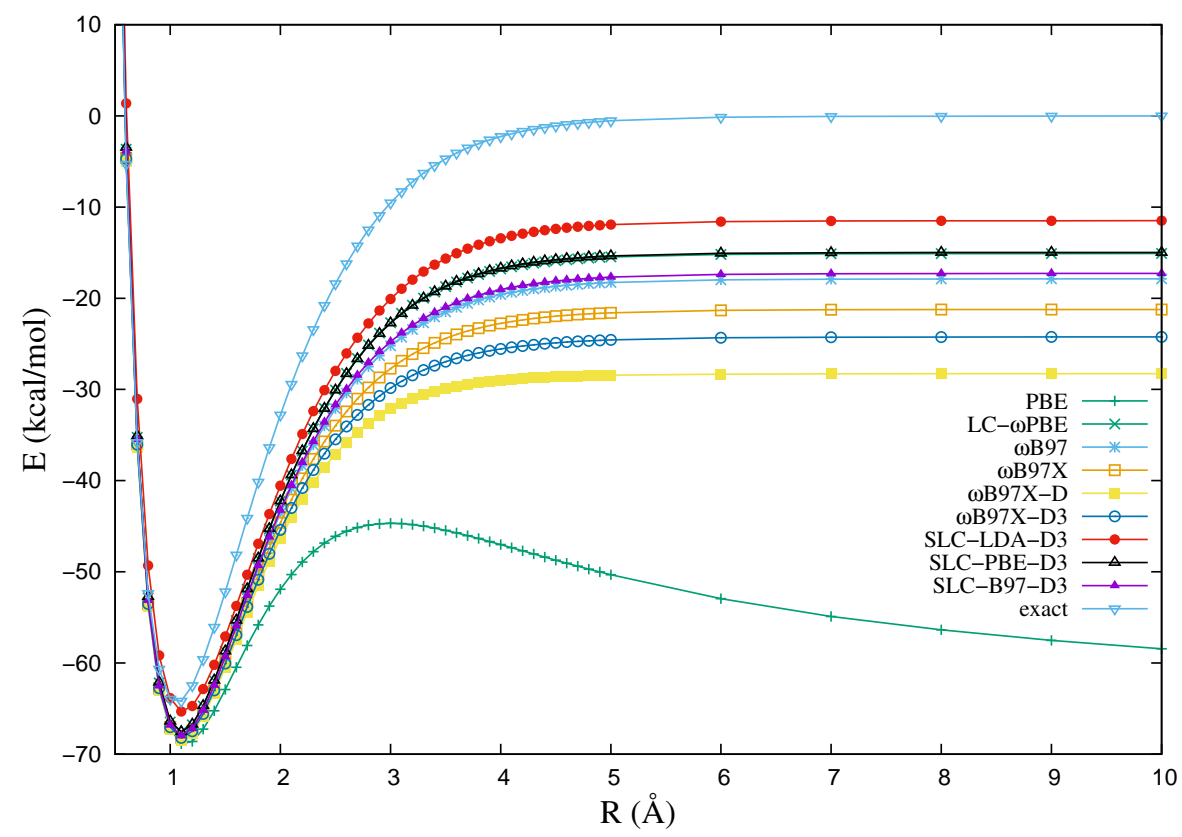

FIG. 2. Dissociation energy curve of $\mathrm{H}_{2}^{+}$. Zero level is set to $E(\mathrm{H})+E\left(\mathrm{H}^{+}\right)$for each method. 


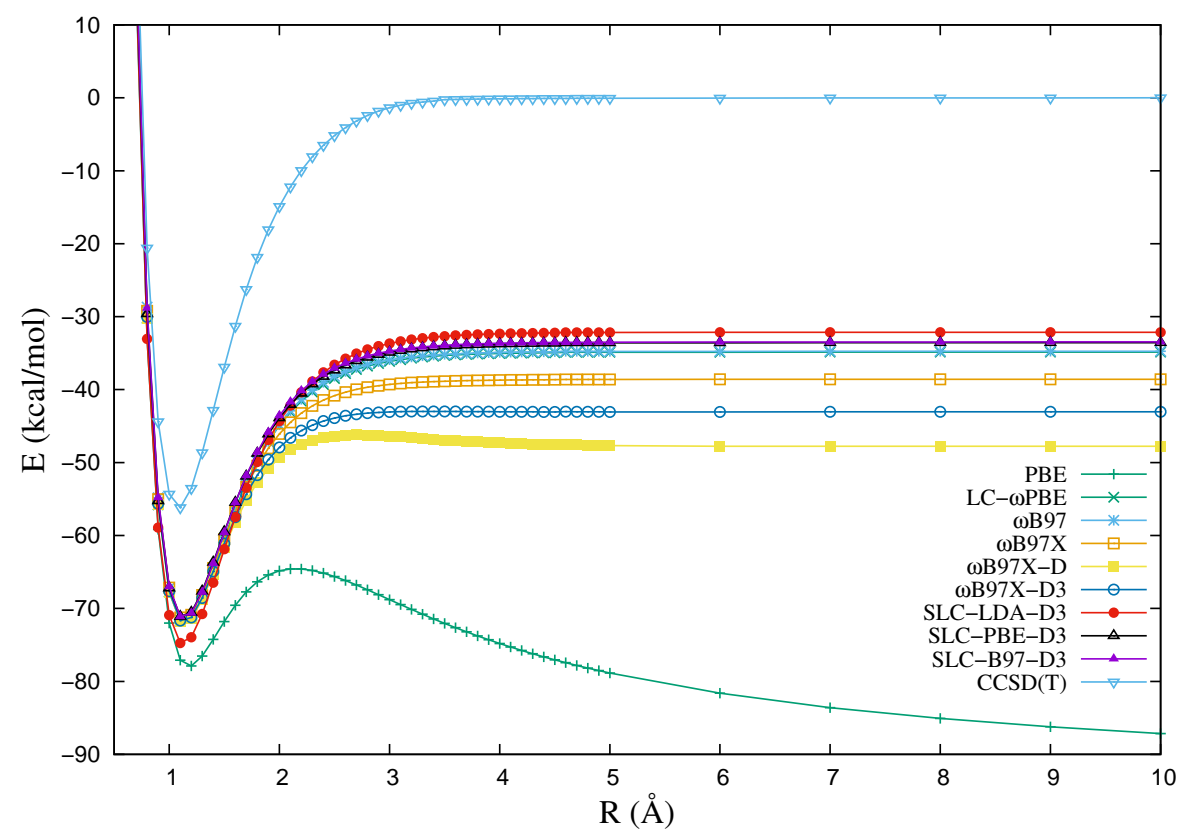

FIG. 3. Dissociation energy curve of $\mathrm{He}_{2}^{+}$. Zero level is set to $E(\mathrm{He})+E\left(\mathrm{He}^{+}\right)$for each method.

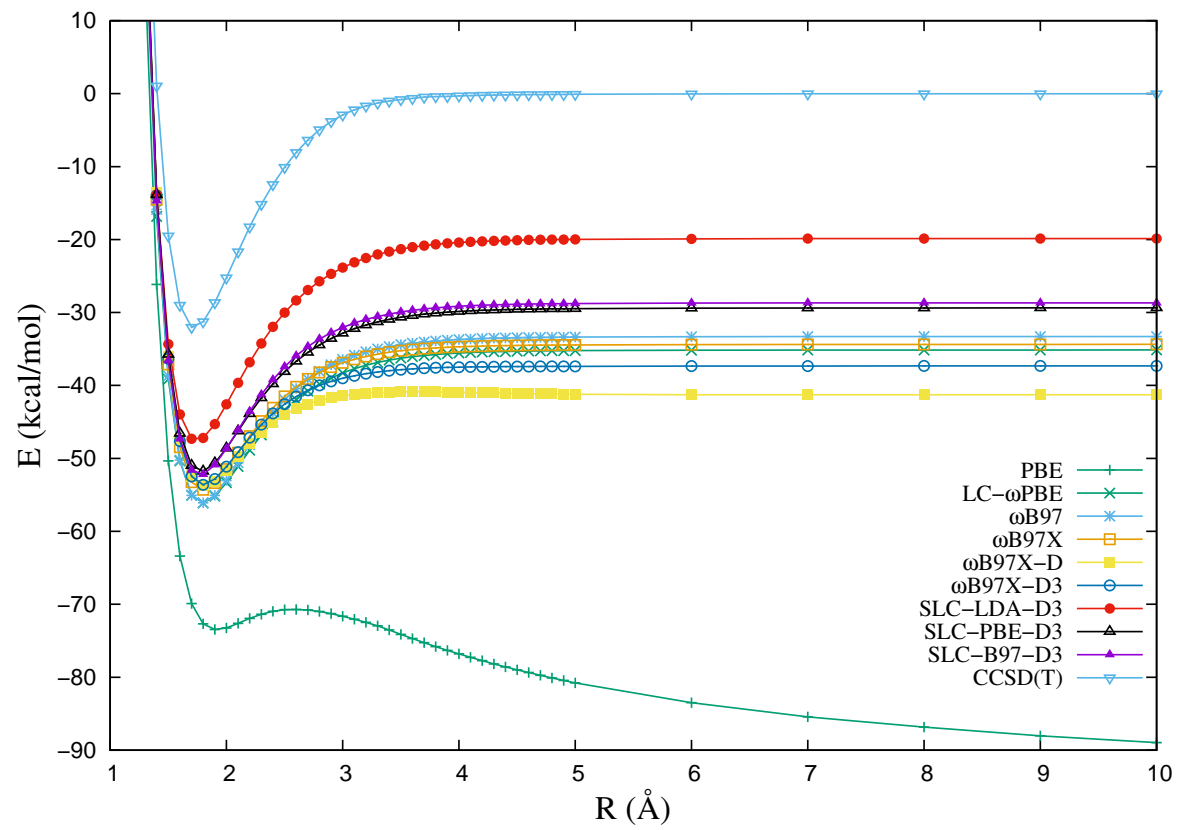

FIG. 4. Dissociation energy curve of $\mathrm{Ne}_{2}^{+}$. Zero level is set to $E(\mathrm{Ne})+E\left(\mathrm{Ne}^{+}\right)$for each method. 


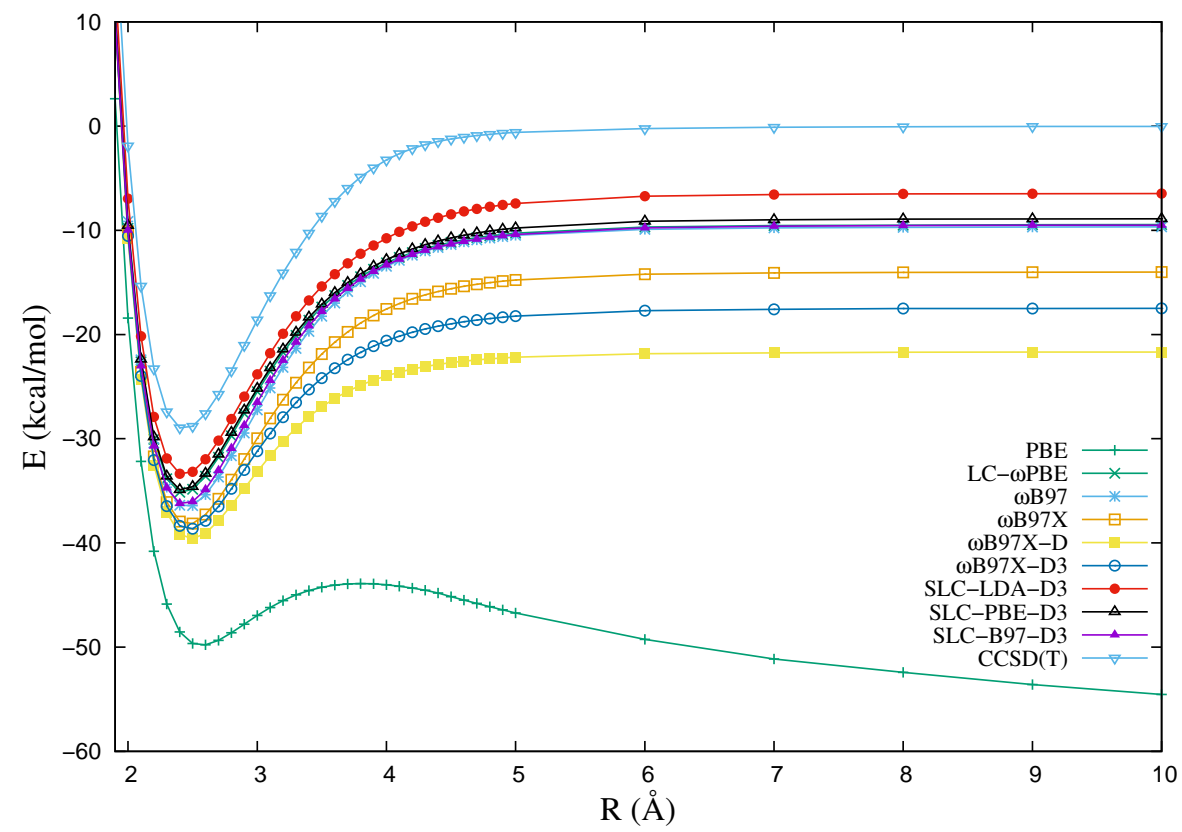

FIG. 5. Dissociation energy curve of $\mathrm{Ar}_{2}^{+}$. Zero level is set to $E(\mathrm{Ar})+E\left(\mathrm{Ar}^{+}\right)$for each method.

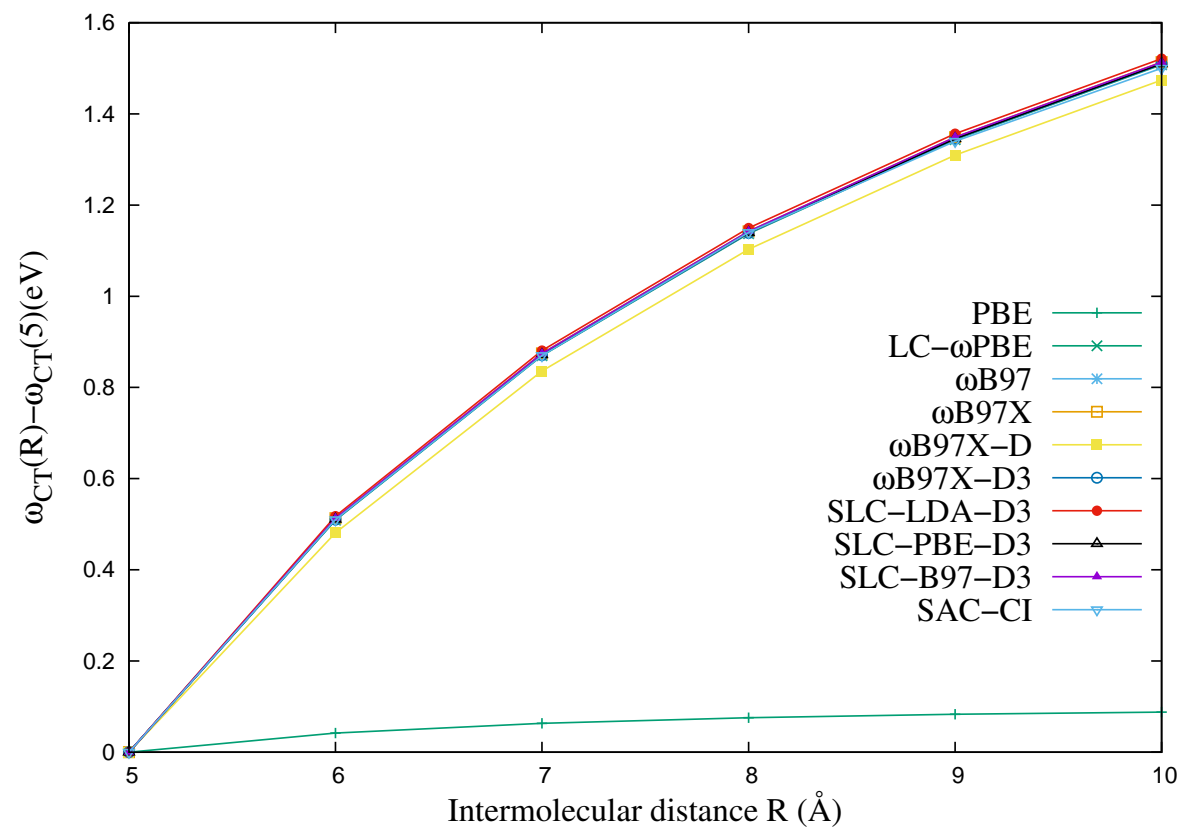

FIG. 6. Relative excitation energy for the lowest CT excitation of $\mathrm{C}_{2} \mathrm{H}_{4} \cdots \mathrm{C}_{2} \mathrm{~F}_{4}$ dimer along the intermolecular distance $R$ (in $\AA$ ). The excitation energy at $5 \AA$ is set to zero for each method. 


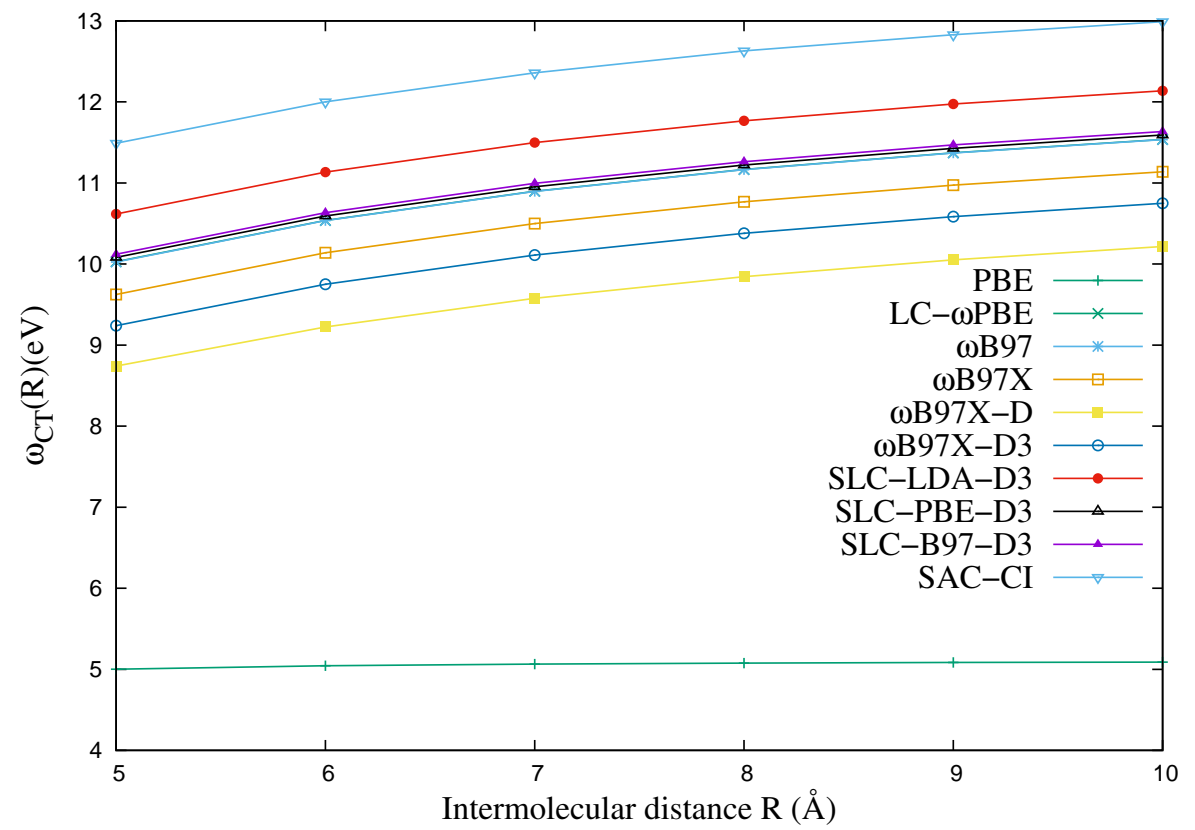

FIG. 7. The lowest CT excitation energy of $\mathrm{C}_{2} \mathrm{H}_{4} \cdots \mathrm{C}_{2} \mathrm{~F}_{4}$ dimer along the intermolecular distance $R$ (in $\AA$ ).

\section{TABLES}

TABLE I. Optimized parameters for SLC-LDA-D3, SLC-PBE-D3, and SLC-B97-D3. Here, $\omega_{\mathrm{SR}}$ and $\omega_{\mathrm{LR}}$ are defined in Equations (5), (11), (15) and (20), $s_{r, 6}$ and $s_{r, 8}$ are defined in Equation (22)), and the others are defined in Equations (18) to (20).

\begin{tabular}{lrrr}
\hline \hline & SLC-B97-D3 & SLC-PBE-D3 & SLC-LDA-D3 \\
\hline$\omega_{\mathrm{SR}}\left(\mathrm{bohr}^{-1}\right)$ & 2.0 & 2.0 & 1.5 \\
$\omega_{\mathrm{LR}}\left(\mathrm{bohr}^{-1}\right)$ & 0.40 & 0.40 & 0.45 \\
$s_{r, 6}$ & 1.298 & 1.179 & 1.129 \\
$s_{r, 8}$ & 1.277 & 1.123 & 1.131 \\
$c_{x \sigma, 0}$ & 1.000000 & & \\
$c_{x \sigma, 1}$ & 1.469313 & & \\
$c_{x \sigma, 2}$ & -6.185202 & &
\end{tabular}




$\begin{array}{lr}c_{x \sigma, 3} & 23.053635 \\ c_{x \sigma, 4} & -16.353923 \\ c_{c \sigma \sigma, 0} & 1.000000 \\ c_{c \sigma \sigma, 1} & -2.154721 \\ c_{c \sigma \sigma, 2} & 10.271378 \\ c_{c \sigma \sigma, 3} & -23.966521 \\ c_{c \sigma \sigma, 4} & 15.345722 \\ c_{c \alpha \beta, 0} & 1.000000 \\ c_{c \alpha \beta, 1} & 4.460711 \\ c_{c \alpha \beta, 2} & -25.043202 \\ c_{c \alpha \beta, 3} & 22.506558 \\ c_{c \alpha \beta, 4} & -4.114590\end{array}$

TABLE II. Statistical errors (in $\mathrm{kcal} / \mathrm{mol}$ ) of the training set. PBE, LC- $\omega$ PBE, and LC- $\omega$ PBE-D3 (statistical errors given in parentheses) were not particularly parametrized using this training set.

\begin{tabular}{|c|c|c|c|c|c|c|c|c|c|c|}
\hline System & Error & PBE & LC- $\omega$ PBE(-D3) & $\omega \mathrm{B} 97$ & $\omega \mathrm{B} 97 \mathrm{X}$ & $\omega \mathrm{B} 97 \mathrm{X}-\mathrm{D}$ & $\omega$ B97X-D3 & SLC-LDA-D3 & SLC-PBE-D3 & SLC-B97-D3 \\
\hline G3/99 & MSE & 20.90 & $3.12(4.79)$ & -0.29 & -0.20 & -0.24 & -0.14 & 2.63 & -0.57 & -0.32 \\
\hline \multirow[t]{2}{*}{$(223)$} & MAE & 21.51 & $5.86(7.17)$ & 2.63 & 2.13 & 1.93 & 2.06 & 8.84 & 4.49 & 2.63 \\
\hline & rms & 26.30 & $7.43(9.02)$ & 3.58 & 2.88 & 2.77 & 2.81 & 11.09 & 5.91 & 3.49 \\
\hline IP & MSE & 0.04 & $2.86(2.85)$ & -0.50 & -0.14 & 0.20 & 0.07 & 11.60 & 1.85 & -0.22 \\
\hline \multirow[t]{2}{*}{$(40)$} & MAE & 3.44 & $4.29(4.29)$ & 2.68 & 2.69 & 2.75 & 2.66 & 11.60 & 3.74 & 2.54 \\
\hline & $\mathrm{rms}$ & 4.35 & $5.39(5.39)$ & 3.60 & 3.59 & 3.62 & 3.53 & 12.40 & 4.70 & 3.45 \\
\hline EA & MSE & 1.72 & $0.18(0.18)$ & 1.52 & -0.47 & 0.07 & -0.37 & 8.96 & -0.54 & -1.66 \\
\hline \multirow[t]{2}{*}{$(25)$} & MAE & 2.42 & $3.00(3.01)$ & 2.72 & 2.04 & 1.91 & 1.93 & 8.96 & 3.05 & 2.66 \\
\hline & $\mathrm{rms}$ & 3.06 & $3.50(3.51)$ & 3.11 & 2.57 & 2.38 & 2.41 & 9.62 & 3.52 & 3.06 \\
\hline PA & MSE & -0.83 & $0.86(0.94)$ & 0.67 & 0.56 & 1.42 & 1.10 & -1.91 & 0.84 & 0.80 \\
\hline \multirow[t]{2}{*}{ (8) } & MAE & 1.60 & $1.41(1.45)$ & 1.48 & 1.21 & 1.50 & 1.29 & 2.31 & 1.36 & 1.44 \\
\hline & $\mathrm{rms}$ & 1.91 & $2.04(2.08)$ & 2.18 & 1.70 & 2.05 & 1.92 & 2.54 & 2.01 & 2.19 \\
\hline NHTBH & MSE & -8.52 & $1.39(1.01)$ & 1.32 & 0.55 & -0.45 & 0.04 & 1.99 & 1.29 & 1.38 \\
\hline \multirow[t]{2}{*}{$(38)$} & MAE & 8.62 & $2.47(2.28)$ & 2.32 & 1.75 & 1.51 & 1.53 & 3.32 & 2.38 & 2.13 \\
\hline & $\mathrm{rms}$ & 10.61 & $3.07(2.83)$ & 2.82 & 2.08 & 2.00 & 1.89 & 3.77 & 2.86 & 2.55 \\
\hline $\mathrm{HTBH}$ & MSE & -9.67 & $-0.77(-1.23)$ & -0.66 & -1.55 & 2.57 & -2.08 & -0.27 & -1.03 & -0.96 \\
\hline \multirow[t]{2}{*}{$(38)$} & MAE & 9.67 & $1.39(1.59)$ & 2.11 & 2.27 & 2.70 & 2.40 & 1.99 & 1.41 & 2.04 \\
\hline & $\mathrm{rms}$ & 10.37 & $1.90(2.07)$ & 2.47 & 2.60 & 3.10 & 2.75 & 2.59 & 1.77 & 2.33 \\
\hline $\mathrm{S} 22$ & MSE & 2.71 & $2.82(-0.08)$ & 0.10 & 0.47 & -0.14 & -0.07 & 0.34 & 0.11 & -0.20 \\
\hline \multirow[t]{2}{*}{$(22)$} & MAE & 2.71 & $2.82(0.26)$ & 0.53 & 0.79 & 0.19 & 0.18 & 0.45 & 0.30 & 0.23 \\
\hline & $\mathrm{rms}$ & 3.73 & $3.58(0.35)$ & 0.63 & 1.11 & 0.25 & 0.25 & 0.61 & 0.39 & 0.33 \\
\hline Total & MSE & 10.32 & $2.30(3.01)$ & -0.23 & -0.21 & -0.38 & -0.28 & 3.38 & -0.12 & -0.26 \\
\hline
\end{tabular}


TABLE III. Statistical errors (in eV) of the 23 core ionization energies of 14 molecules taken from Ref. [135]. The relativistic corrections are not considered.

\begin{tabular}{lrrrrrrrrrr}
\hline \hline System & Error & PBE & LC- $\omega$ PBE & $\omega$ B97 & $\omega$ B97X & $\omega$ B97X-D & $\omega$ B97X-D3 & SLC-LDA-D3 & SLC-PBE-D3 & SLC-B97-D3 \\
\hline Core & MSE & -26.25 & -20.15 & -19.39 & -14.88 & -13.74 & -14.10 & 4.25 & -2.36 & -1.53 \\
Ionization & MAE & 26.25 & 20.15 & 19.39 & 14.88 & 13.74 & 14.10 & 4.27 & 2.77 & 2.53 \\
$(23)$ & rms & 26.48 & 20.47 & 19.70 & 15.10 & 13.91 & 14.29 & 5.27 & 3.36 & 2.91 \\
\hline \hline
\end{tabular}

TABLE IV. Statistical errors (in eV) of the 38 core excitation energies of 13 molecules taken from Ref. [114]. The relativistic corrections are not considered.

\begin{tabular}{lrrrrrrrrrr}
\hline \hline State & Error & PBE & LC- $\omega$ PBE & $\omega$ B97 & $\omega$ B97X & $\omega$ B97X-D & $\omega$ B97X-D3 & SLC-LDA-D3 & SLC-PBE-D3 & SLC-B97-D3 \\
\hline Core $\rightarrow$ & MSE & -42.32 & -41.30 & -40.31 & -31.95 & -28.74 & -30.05 & 4.81 & -1.46 & -0.38 \\
Valence & MAE & 42.32 & 41.30 & 40.31 & 31.95 & 28.74 & 30.05 & 5.12 & 2.22 & 2.53 \\
$(15)$ & rms & 50.91 & 49.93 & 48.96 & 39.29 & 35.56 & 37.08 & 6.25 & 2.91 & 2.84 \\
Core $\rightarrow$ & MSE & -32.26 & -29.35 & -28.38 & -22.26 & -20.35 & -21.12 & 3.36 & -2.93 & -1.93 \\
Rydberg & MAE & 32.26 & 29.35 & 28.38 & 22.26 & 20.35 & 21.12 & 3.50 & 3.22 & 2.94 \\
$(23)$ & rms & 39.91 & 37.64 & 36.78 & 29.43 & 26.90 & 27.93 & 4.94 & 3.81 & 3.26 \\
\hline \hline
\end{tabular}

TABLE V. Statistical errors (in $\mathrm{kcal} / \mathrm{mol}$ ) of the S66 set [136].

\begin{tabular}{lrrrrrrrrrr}
\hline \hline System & Error & PBE & LC- $\omega$ PBE & $\omega$ B97 & $\omega$ B97X & $\omega$ B97X-D & $\omega$ B97X-D3 & SLC-LDA-D3 & SLC-PBE-D3 & SLC-B97-D3 \\
\hline S66 & MSE & 2.22 & 2.46 & -0.15 & 0.16 & -0.30 & -0.23 & 0.04 & -0.06 & -0.35 \\
$(66)$ & MAE & 2.23 & 2.46 & 0.37 & 0.49 & 0.35 & 0.26 & 0.21 & 0.27 & 0.37 \\
& rms & 2.75 & 2.80 & 0.47 & 0.65 & 0.51 & 0.35 & 0.30 & 0.35 & 0.46 \\
\hline \hline
\end{tabular}

TABLE VI. Statistical errors (in eV) of the AE113 database [52, 84].

\begin{tabular}{lrrrrrrrrrr}
\hline \hline System & Error & PBE & LC- $\omega$ PBE & $\omega$ B97 & $\omega$ B97X & $\omega$ B97X-D & $\omega$ B97X-D3 & SLC-LDA-D3 & SLC-PBE-D3 & SLC-B97-D3 \\
\hline AE113 & MSE & 0.83 & 0.10 & 0.05 & 0.05 & 0.04 & 0.05 & 0.04 & -0.03 & 0.04 \\
$(113)$ & MAE & 0.88 & 0.27 & 0.11 & 0.10 & 0.10 & 0.10 & 0.28 & 0.17 & 0.11 \\
& rms & 1.06 & 0.41 & 0.15 & 0.13 & 0.14 & 0.13 & 0.34 & 0.23 & 0.14 \\
\hline \hline
\end{tabular}

TABLE VII. Statistical errors (in eV) of the IP131 database [84]. 


\begin{tabular}{|c|c|c|c|c|c|c|c|c|c|c|}
\hline System & Error & PBE & $\mathrm{LC}-\omega \mathrm{PBE}$ & $\omega \mathrm{B} 97$ & $\omega \mathrm{B} 97 \mathrm{X}$ & $\omega \mathrm{B} 97 \mathrm{X}-\mathrm{D}$ & $\omega \mathrm{B} 97 \mathrm{X}-\mathrm{D} 3$ & SLC-LDA-D3 & SLC-PBE-D3 & SLC-B97-D3 \\
\hline \multicolumn{11}{|c|}{$\operatorname{IP}(1)=E_{\text {total }}(N-1)-E_{\text {total }}(N)$} \\
\hline IP131 & MSE & -0.26 & 0.10 & 0.00 & 0.00 & -0.03 & -0.02 & 0.57 & 0.09 & 0.02 \\
\hline \multirow[t]{2}{*}{$(131)$} & MAE & 0.36 & 0.28 & 0.19 & 0.18 & 0.19 & 0.18 & 0.58 & 0.20 & 0.18 \\
\hline & $\mathrm{rms}$ & 0.52 & 0.46 & 0.26 & 0.26 & 0.27 & 0.26 & 0.64 & 0.28 & 0.26 \\
\hline \multicolumn{11}{|c|}{$\operatorname{IP}(2)=-\epsilon_{\mathrm{HOMO}}(N)$} \\
\hline IP131 & MSE & -4.40 & -0.15 & -0.24 & -0.48 & -1.01 & -0.71 & 0.61 & -0.09 & -0.18 \\
\hline \multirow[t]{2}{*}{$(131)$} & MAE & 4.40 & 0.42 & 0.40 & 0.51 & 1.01 & 0.72 & 0.70 & 0.36 & 0.37 \\
\hline & rms & 4.50 & 0.68 & 0.63 & 0.75 & 1.18 & 0.93 & 0.77 & 0.56 & 0.59 \\
\hline
\end{tabular}

TABLE VIII. Statistical errors (in eV) of the EA131 database [52, 84].

\begin{tabular}{|c|c|c|c|c|c|c|c|c|c|c|}
\hline System & Error & $\mathrm{PBE}$ & $\mathrm{LC}-\omega \mathrm{PBE}$ & $\omega \mathrm{B} 97$ & $\omega \mathrm{B} 97 \mathrm{X}$ & $\omega \mathrm{B} 97 \mathrm{X}-\mathrm{D}$ & $\omega \mathrm{B} 97 \mathrm{X}-\mathrm{D} 3$ & SLC-LDA-D3 & SLC-PBE-D3 & SLC-B97-D3 \\
\hline \multicolumn{11}{|c|}{$\mathrm{EA}(1)=E_{\text {total }}(N)-E_{\text {total }}(N+1)$} \\
\hline EA131 & MSE & 0.10 & -0.11 & -0.24 & -0.19 & -0.14 & -0.16 & 0.23 & -0.08 & -0.22 \\
\hline \multirow[t]{2}{*}{$(131)$} & MAE & 0.21 & 0.36 & 0.34 & 0.31 & 0.26 & 0.29 & 0.33 & 0.27 & 0.32 \\
\hline & $\mathrm{rms}$ & 0.34 & 0.54 & 0.44 & 0.41 & 0.36 & 0.39 & 0.45 & 0.35 & 0.42 \\
\hline \multicolumn{11}{|c|}{$\mathrm{EA}(2)=-\epsilon_{\mathrm{HOMO}}(N+1)$} \\
\hline EA131 & MSE & -2.03 & 0.00 & -0.13 & -0.17 & -0.32 & -0.23 & 0.40 & 0.08 & -0.10 \\
\hline \multirow[t]{2}{*}{$(131)$} & MAE & 2.03 & 0.34 & 0.34 & 0.32 & 0.39 & 0.33 & 0.47 & 0.30 & 0.33 \\
\hline & $\mathrm{rms}$ & 2.30 & 0.43 & 0.43 & 0.41 & 0.51 & 0.44 & 0.62 & 0.38 & 0.41 \\
\hline \multicolumn{11}{|c|}{$\operatorname{EA}(3)=-\epsilon_{\mathrm{LUMO}}(N)$} \\
\hline EA131 & MSE & 2.43 & -0.19 & -0.38 & -0.31 & 0.01 & -0.15 & 0.10 & -0.25 & -0.34 \\
\hline \multirow[t]{2}{*}{ (131) } & MAE & 2.45 & 0.42 & 0.49 & 0.52 & 0.54 & 0.49 & 0.30 & 0.39 & 0.44 \\
\hline & $\mathrm{rms}$ & 2.72 & 0.51 & 0.59 & 0.62 & 0.63 & 0.57 & 0.40 & 0.47 & 0.53 \\
\hline
\end{tabular}

TABLE IX. Statistical errors (in eV) of the FG131 database [52, 84].

\begin{tabular}{|c|c|c|c|c|c|c|c|c|c|c|}
\hline System & Error & PBE & $\mathrm{LC}-\omega \mathrm{PBE}$ & $\omega \mathrm{B} 97$ & $\omega \mathrm{B} 97 \mathrm{X}$ & $\omega \mathrm{B} 97 \mathrm{X}-\mathrm{D}$ & $\omega \mathrm{B} 97 \mathrm{X}-\mathrm{D} 3$ & SLC-LDA-D3 & SLC-PBE-D3 & SLC-B97-D3 \\
\hline \multicolumn{11}{|c|}{$E_{g}(1)=E_{\text {total }}(N-1)+E_{\text {total }}(N+1)-2 E_{\text {total }}(N)$} \\
\hline FG131 & MSE & -0.46 & 0.10 & 0.13 & 0.08 & -0.01 & 0.03 & 0.23 & 0.05 & 0.13 \\
\hline \multirow[t]{2}{*}{$(131)$} & MAE & 0.57 & 0.44 & 0.32 & 0.29 & 0.27 & 0.28 & 0.39 & 0.30 & 0.32 \\
\hline & $\mathrm{rms}$ & 0.76 & 0.74 & 0.42 & 0.40 & 0.38 & 0.39 & 0.47 & 0.39 & 0.41 \\
\hline \multicolumn{11}{|c|}{$E_{g}(2)=\epsilon_{\mathrm{HOMO}}(N+1)-\epsilon_{\mathrm{HOMO}}(N)$} \\
\hline FG131 & MSE & -2.48 & -0.26 & -0.22 & -0.42 & -0.80 & -0.59 & 0.09 & -0.27 & -0.18 \\
\hline \multirow[t]{2}{*}{$(131)$} & MAE & 2.48 & 0.50 & 0.44 & 0.50 & 0.81 & 0.62 & 0.47 & 0.43 & 0.43 \\
\hline & $\mathrm{rms}$ & 2.69 & 0.70 & 0.59 & 0.65 & 0.93 & 0.77 & 0.57 & 0.57 & 0.57 \\
\hline \multicolumn{11}{|c|}{$E_{g}(3)=\epsilon_{\mathrm{LUMO}}(N)-\epsilon_{\mathrm{HOMO}}(N)$} \\
\hline FG131 & MSE & -6.94 & -0.07 & 0.04 & -0.27 & -1.12 & -0.67 & 0.40 & 0.05 & 0.05 \\
\hline \multirow[t]{2}{*}{ (131) } & MAE & 6.94 & 0.53 & 0.49 & 0.57 & 1.15 & 0.77 & 0.54 & 0.45 & 0.45 \\
\hline & $\mathrm{rms}$ & 7.15 & 0.80 & 0.65 & 0.77 & 1.40 & 1.02 & 0.63 & 0.59 & 0.60 \\
\hline
\end{tabular}


TABLE X. Statistical errors (in eV) of the 19 valence and 23 Rydberg excitation energies of five molecules $\left(\mathrm{N}_{2}, \mathrm{CO}\right.$, water, ethylene, and formaldehyde) taken from Ref. [137].

\begin{tabular}{lrrrrrrrrrr}
\hline \hline State & Error & PBE & LC- $\omega$ PBE & $\omega$ B97 & $\omega$ B97X & $\omega$ B97X-D & $\omega$ B97X-D3 & SLC-LDA-D3 & SLC-PBE-D3 & SLC-B97-D3 \\
\hline Valence & MSE & -0.30 & -0.36 & -0.23 & -0.28 & -0.29 & -0.28 & -0.32 & -0.37 & -0.23 \\
$(19)$ & MAE & 0.31 & 0.39 & 0.27 & 0.30 & 0.30 & 0.29 & 0.37 & 0.40 & 0.27 \\
& rms & 0.38 & 0.47 & 0.34 & 0.37 & 0.37 & 0.37 & 0.46 & 0.48 & 0.35 \\
Rydberg & MSE & -1.29 & 0.19 & 0.24 & 0.12 & -0.30 & -0.12 & 0.51 & 0.20 & 0.22 \\
$(23)$ & MAE & 1.29 & 0.29 & 0.28 & 0.21 & 0.35 & 0.22 & 0.51 & 0.28 & 0.27 \\
& rms & 1.35 & 0.38 & 0.37 & 0.30 & 0.40 & 0.30 & 0.58 & 0.37 & 0.36 \\
\hline \hline
\end{tabular}

